\title{
The entropy of Nakada's $\alpha$-continued fractions: analytical results
}

\author{
GiUlio Tiozzo
}

\begin{abstract}
We study the ergodic theory of a one-parameter family of interval maps $T_{\alpha}$ arising from generalized continued fraction algorithms. First of all, we prove the dependence of the metric entropy of $T_{\alpha}$ to be Hölder-continuous in the parameter $\alpha$. Moreover, we prove a central limit theorem for possibly unbounded observables whose bounded variation grows moderately. This class of functions is large enough to cover the case of Birkhoff averages converging to the entropy.
\end{abstract}

Mathematics Subject Classification (2010): 11K50 (primary); 37A10, 37A55, 37E05 (secondary).

\section{Introduction}

Let $\alpha \in[0,1]$. Let us define the map $T_{\alpha}:[\alpha-1, \alpha] \rightarrow[\alpha-1, \alpha]$ as $T_{\alpha}(0)=0$ and

$$
T_{\alpha}(x)=\frac{1}{|x|}-a_{\alpha}(x)
$$

with $a_{\alpha}(x):=\left\lfloor\frac{1}{|x|}+1-\alpha\right\rfloor$. These systems were introduced by Nakada [13] and are known in the literature as $\alpha$-continued fractions, or Japanese continued fractions. By taking $x_{n, \alpha}=T_{\alpha}^{n}(x), a_{n, \alpha}=a_{\alpha}\left(x_{n-1, \alpha}\right), \epsilon_{n, \alpha}=\operatorname{Sign}\left(x_{n-1, \alpha}\right)$, the orbit under $T_{\alpha}$ generates the generalized continued fraction expansion

$$
x=a_{0, \alpha}+\frac{\epsilon_{1, \alpha}}{a_{1, \alpha}+\frac{\epsilon_{2, \alpha}}{a_{2, \alpha}+} \cdot} .
$$

The algorithm, analogously to the Gauss map in the classical case, provides rational approximations of real numbers. The family $T_{\alpha}$ interpolates between well-known continued fraction algorithms: namely, $T_{1}$ is the usual Gauss map, while $T_{1 / 2}$ generates the continued fraction to nearest integer, and $T_{0}$ generates the backward or by-excess continued fraction expansion (see [17] for a general reference).

Received October 28, 2011; accepted in revised form September 13, 2012. 
It is known that for each $\alpha \in(0,1]$ there exists a unique invariant measure $\mu_{\alpha}(d x)=\rho_{\alpha}(x) d x$ absolutely continuous with respect to the Lebesgue measure, and this measure is ergodic (see [11]). On the other hand, the map $T_{0}$ has an indifferent fixed point, and only admits an infinite invariant measure.

In this paper we will focus on the metric entropy of the $T_{\alpha}$ 's, which is given by Rohlin's formula (see [15])

$$
h\left(T_{\alpha}\right)=\int_{\alpha-1}^{\alpha} \log \left|T_{\alpha}^{\prime}\right| d \mu_{\alpha} .
$$

Nakada [13] computed exact values of $h\left(T_{\alpha}\right)$ for $\alpha \geq \frac{1}{2}$, showing that in this interval $h\left(T_{\alpha}\right)$ is continuous, and smooth except for the point $\alpha=\frac{\sqrt{5}-1}{2}$, where the left and right derivatives do not coincide. Cassa, Moussa and Marmi [12] computed the exact value of the invariant density, hence the entropy, for $\alpha \in(\sqrt{2}-1,1 / 2)$.

In [11], Luzzi and Marmi studied the behaviour of $h\left(T_{\alpha}\right)$ as a function of $\alpha$ for all parameters $\alpha \in(0,1]$. They gave numerical evidence that this function is continuous, but not smooth, and tends to zero as $\alpha$ tends to zero.

Nakada and Natsui [14] then proved that $h\left(T_{\alpha}\right)$ is of the order of $\frac{-1}{\log \alpha}$ when $\alpha$ is near 0 , and that the entropy is not monotone. Indeed, they explicitely constructed infinitely many intervals over which $h\left(T_{\alpha}\right)$ is monotone, but the monotonicity varies according to the interval and can be increasing, decreasing, or constant.

An extensive numerical study of these intervals has been carried out in [2], and a complete characterization of all monotonicity intervals is given in [3]. Even though the entropy is conjecturally smooth on any such interval, there are points at which it is not even locally monotone, and the set of bifurcation parameters has a complicated self-similar structure [4].

The first major result of this paper is the following:

Theorem 1.1. The entropy function $\alpha \mapsto h\left(T_{\alpha}\right)$ is Hölder-continuous of any exponent $s$ for $0<s<\frac{1}{2}$.

The proof follows from spectral analysis of the transfer operator acting on the space of functions of bounded variation. First (Section 3.1), we prove a uniform bound on the essential spectral radius (Lasota-Yorke inequality). Then (Section 3.2 ), we prove that a suitable distance between the transformations $T_{\alpha}$ is Höldercontinuous in $\alpha$, and we use a stability result of the spectral decomposition [8] to prove Hölder-continuity of the invariant densities $\rho_{\alpha}$ in $L^{1}$-norm. Note that invariant densities are not continuous in $B V$-norm (see Remark 3.11).

The second part of the paper deals with central limit theorems. In [11], the entropy is computed by approximating it with Birkhoff averages for the observable $\log \left|T_{\alpha}^{\prime}\right|$, and numerical evidence is given [11, Figure 3] that Birkhoff sums for different orbits distribute normally around the average. We first show (Section 4.1) that the methods of [1] can be used to prove a central limit theorem for observables of bounded variation. 
Moreover, in Section 4.2 we expand the class of observables we use in order to encompass unbounded observables such as the logarithm. Indeed, we will define a new family of Banach spaces $B_{K, \delta}$, consisting of possibly unbounded functions whose total variation grows slowly on intervals which approach zero. Such functions will be called of mild growth, and we prove the central limit theorem to hold in these larger spaces:

Theorem 1.2. Take $\alpha \in(0,1], 0<\delta<\frac{1}{2}$, and $K$ sufficiently large. Then for every non-constant real-valued $f \in B_{K, \delta}$ there exists $\sigma>0$ such that

$$
\lim _{n \rightarrow \infty} \mu_{\alpha}\left(\frac{S_{n}\left(f-\int_{I_{\alpha}} f d \mu_{\alpha}\right)}{\sqrt{n}} \leq v\right)=\frac{1}{\sigma \sqrt{2 \pi}} \int_{v}^{+\infty} e^{-\frac{t^{2}}{2 \sigma^{2}}} d t \quad \forall v \in \mathbb{R} .
$$

As a corollary, Birkhoff sums for the observable $\log \left|T_{\alpha}^{\prime}\right|$ distribute normally around the average value $h\left(T_{\alpha}\right)$

Finally, in Section 5 we discuss the dependence of the standard deviation of Birkhoff averages on the parameter $\alpha$. More precisely, given some observable $f$ of class $C^{1}$, for which we proved the central limit theorem to hold, we will prove that the variance $\sigma_{\alpha, f}^{2}$ of the limit Gaussian distribution is continuous in $\alpha$. The result is motivated by numerical data in [2, Section 2.3].

Many different authors have studied the spectral properties of transfer operators of expanding maps. For instance, a spectral decomposition for individual expanding maps is proved in $[1,16]$ and [18]. A brief historical account with references is given in [8]. In our case, however, it is essential to prove estimates on the spectral radius which are uniform in $\alpha$. Since new branches of $T_{\alpha}$ appear as $\alpha$ moves, and $T_{\alpha}$ develops an indifferent fixed point as $\alpha \rightarrow 0$, proving uniformity requires more work. Unfortunately, although a uniform Lasota-Yorke inequality holds, the proof provided by [11] contains a bug; we shall therefore produce a new proof in Proposition 3.1. Another proof of continuity (not Hölder) of entropy is given in the very recent paper [10] via a study of natural extensions. We do not know whether our estimate on the Hölder exponent is sharp; however, we have strong reasons to believe that the entropy function is not locally Lipschitz (see the end of Section 3.3).

Let us finally remark that our functional-analytic methods only use a few properties of $T_{\alpha}$, hence they can be applied to a wider class of one-parameter families of expanding interval maps. For instance, they apply to the case of the $(a, b)$-continued fraction transformations studied in [9] for parameters on the critical line $b-a=1$.

\section{Basic properties}

Let us start by setting up the framework needed for the rest of the paper, and establishing a basic spectral decomposition for the transfer operator. The literature on thermodynamic formalism for interval maps is huge: the sources we mainly refer to are $[1,16]$ and $[18]$, which already make use of functions of bounded variation. 
The total variation of a function $f$ on a set $X \subseteq \mathbb{R}$ is

$$
\operatorname{Var}_{X} f:=\sup \sum_{i=1}^{n}\left|f\left(x_{i}\right)-f\left(x_{i+1}\right)\right|
$$

where the sup is taken over all finite increasing sequences $x_{1} \leq x_{2} \leq \cdots \leq x_{n}$ of points of $X$. Given an interval $I$, let us denote $B V(I)$ the Banach space of complexvalued bounded variation functions of the interval $I$, modulo equality almost everywhere. The space is endowed with the norm

$$
\|f\|_{B V(I)}:=\inf \left\{\operatorname{Var}_{I} g+\int_{I}|g(x)| d x: g=f \text { a.e. }\right\} .
$$

Observe that every $f \in B V(I)$ has a (not necessarily unique) representative of minimal total variation, namely such that

$$
f(x) \in\left[\lim _{y \rightarrow x^{-}} f(y), \lim _{y \rightarrow x^{+}} f(y)\right] \quad \forall x \in I .
$$

In the following, we will always choose representatives for our functions of minimal variation. Other basic properties of total variation are stated in the Appendix.

\subsection{Cylinders}

For each $\alpha \in(0,1)$, the dynamical system $T_{\alpha}$ defined in the introduction acts on the interval $I_{\alpha}:=[\alpha-1, \alpha]$.

Observe that there exists a partition of $I_{\alpha}$ in a countable number of intervals $I_{j}$ such that for every $j$ the restriction $T_{\alpha} I_{j}$ is a strictly monotone, $C^{\infty}$ function and it extends to a $C^{\infty}$ function on the closure of every $I_{j}$. The least fine of such partitions will be called $\mathcal{P}_{1}$, the partition associated to $T_{\alpha}$.

Dynamical systems possessing such a partition are sometimes known as fibred systems, and their dynamics can be understood by keeping track of which elements of the partition are visited along the orbits, i.e. in terms of symbolic dynamics. For a general introduction to fibred systems and their applications to number theory, see [17].

More specifically, in our case we have $\mathcal{P}_{1}=\left\{I_{j}^{+}\right\}_{j \geq j_{\min }} \cup\left\{I_{j}^{-}\right\}_{j \geq 2}$ with $j_{\min }=$ $\left\lceil\frac{1}{\alpha}-\alpha\right\rceil$ where

$$
\begin{aligned}
& I_{j}^{+}=\left(\frac{1}{j+\alpha}, \frac{1}{j-1+\alpha}\right) \text { if } j \geq j_{\min }+1 \quad I_{j_{\min }}^{+}=\left(\frac{1}{j_{\min }+\alpha}, \alpha\right) \\
& I_{j}^{-}=\left(-\frac{1}{j-1+\alpha},-\frac{1}{j+\alpha}\right) \text { if } j \geq 3 \quad I_{2}^{-}=\left(\alpha-1, \frac{1}{2+\alpha}\right) .
\end{aligned}
$$

Moreover, for every $n>1$, the set

$$
\left\{I_{j_{1}}^{\epsilon_{1}} \cap T_{\alpha}^{-1}\left(I_{j_{2}}^{\epsilon_{2}}\right) \cap \cdots \cap T^{-(n-1)}\left(I_{j_{n}}^{\epsilon_{n}}\right): I_{j_{1}}^{\epsilon_{1}}, \ldots, I_{j_{n}}^{\epsilon_{n}} \in \mathcal{P}_{1}\right\}
$$


where $\epsilon_{i} \in\{+,-\}$, is a partition of $I_{\alpha}$ in a countable number of intervals such that on each of these the restriction of $T_{\alpha}^{n}$ is monotone and $C^{\infty}$ : such a partition will be denoted by $\mathcal{P}_{n}$ and its elements called cylinders. The cylinder $I_{j_{1}}^{\epsilon_{1}} \cap T_{\alpha}^{-1}\left(I_{j_{2}}^{\epsilon_{2}}\right) \cap \cdots \cap$ $T^{-(n-1)}\left(I_{j_{n}}^{\epsilon_{n}}\right)$ will be denoted either by $\left(I_{j_{1}}^{\epsilon_{1}}, \ldots, I_{j_{n}}^{\epsilon_{n}}\right)$ or by $\left(\left(j_{1}, \epsilon_{1}\right), \ldots,\left(j_{n}, \epsilon_{n}\right)\right)$. The cylinders $I_{j} \in \mathcal{P}_{n}$ such that $T_{\alpha}^{n}\left(I_{j}\right)=I_{\alpha}$ will be called full cylinders.

Let us define the function

$$
g_{n, \alpha}(x):=\sum_{j \in \mathcal{P}_{n}} \frac{1}{\left|\left(T_{\alpha}^{n}\right)^{\prime}(x)\right|} \chi_{I_{j}}(x) .
$$

The following estimates, proven in the Appendix, will be used throughout the paper:

Proposition 2.1. For every $\alpha \in(0,1)$ and for every $n \geq 1$

1. $\left\|g_{n, \alpha}\right\|_{\infty} \leq \gamma_{\alpha}^{n}$ where $\gamma_{\alpha}:=\max \left\{\alpha^{2},(\alpha-1)^{2}\right\}$;

2. $\sup _{j \in \mathcal{P}_{n}} \sup _{x \in I_{j}}\left|g_{n, \alpha}^{\prime}(x)\right| \leq \frac{2}{1-\gamma_{\alpha}}$;

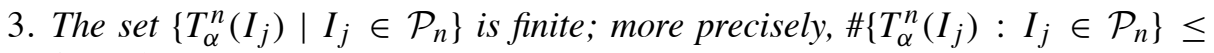
$2 n+1$;

4. The total variation of $g_{1, \alpha}$ is universally bounded, i.e., there is a constant $C_{0}$ such that

$$
\operatorname{Var}_{I_{\alpha}} g_{1, \alpha} \leq C_{0}<+\infty \quad \forall \alpha \in(0,1)
$$

\subsection{Spectral decomposition}

The transfer operator (also known as Ruelle-Perron-Frobenius operator) $\Phi_{\alpha}$ : $L^{1}\left(I_{\alpha}\right) \rightarrow L^{1}\left(I_{\alpha}\right)$ is defined via the duality

$$
\int_{I_{\alpha}} \Phi_{\alpha}(f) g d x=\int_{I_{\alpha}} f\left(g \circ T_{\alpha}\right) d x \quad \forall f \in L^{\infty}\left(I_{\alpha}\right) .
$$

Let us recall that the $n^{\text {th }}$ iterate of the transfer operator is given by

$$
\Phi_{\alpha}^{n}(f)=\sum_{j \in \mathcal{P}_{n}} \frac{f \circ \sigma_{j}}{\left|\left(T_{\alpha}^{n}\right)^{\prime} \circ \sigma_{j}\right|} \chi_{T_{\alpha}^{n}\left(I_{j}\right)}
$$

where $\sigma_{j}: T_{\alpha}^{n}\left(I_{j}\right) \rightarrow I_{j}$ is the inverse of the restriction $\left.T_{\alpha}^{n}\right|_{I_{j}}: I_{j} \rightarrow T_{\alpha}^{n}\left(I_{j}\right)$.

Even though $\Phi_{\alpha}$ is so far defined on $L^{1}$, it turns out that the transfer operator preserves the subspace $B V\left(I_{\alpha}\right)$, and indeed it has good convergence properties in 
$B V$-norm. More precisely, we can now prove the following:

Theorem 2.2. Let $\Phi_{\alpha}: L^{1}\left(I_{\alpha}\right) \rightarrow L^{1}\left(I_{\alpha}\right)$ be the transfer operator for the system $T_{\alpha}$, with $\alpha \in(0,1)$. Then one can write

$$
\Phi_{\alpha}=\Pi_{\alpha}+\Psi_{\alpha}
$$

where $\Pi_{\alpha}$ and $\Psi_{\alpha}$ are commuting, linear bounded operators on $B V\left(I_{\alpha}\right)$. Moreover, $\Psi_{\alpha}$ is a linear bounded operator on $B V\left(I_{\alpha}\right)$ of spectral radius strictly less than 1 , and $\Pi_{\alpha}$ is a projector onto the one-dimensional eigenspace relative to the eigenvalue 1. It is given by

$$
\Pi_{\alpha}(f)=\lim _{n \rightarrow \infty} \frac{1}{n} \sum_{k=1}^{n} \Phi_{\alpha}^{k}(f)
$$

where the convergence is in $L^{1}$.

Corollary 2.3. For every $\alpha \in(0,1), T_{\alpha}$ has exactly one invariant probability measure $\mu_{\alpha}$ which is absolutely continuous with respect to Lebesgue measure. Its density will be denoted by $\rho_{\alpha}$.

Proof. Let us fix $\alpha \in(0,1)$. By Proposition 2.1, we can apply [1, Proposition 4.1], which yields via Ionescu-Tulcea and Marinescu's theorem [6] the following spectral decomposition

$$
\Phi_{\alpha}=\sum_{i=0}^{p} \lambda_{i} \Phi_{i}+\Psi_{\alpha}
$$

where $\left|\lambda_{i}\right|=1$, and the $\Phi_{i}$ are linear bounded operators on $B V\left(I_{\alpha}\right)$ with finitedimensional image, and $\rho\left(\Psi_{\alpha}\right)<1$. Now, it is known [11, Lemma 1] that $T_{\alpha}$ is exact in Rohlin's sense (see [15]); this implies that the invariant measure we have found is ergodic and mixing, which in turn implies that the only eigenvalue of $\Phi_{\alpha}$ of modulus 1 is 1 itself and that its associated eigenspace is one-dimensional (see [18, Chapter 3]).

The spectral decomposition also immediately implies the following exponential decay of correlations:

Proposition 2.4. For any $\alpha \in(0,1)$ there exist $C, \lambda, 0<\lambda<1$ such that for every $n \in \mathbb{N}$ and for every $f_{1}, f_{2} \in B V\left(I_{\alpha}\right)$

$$
\left|\int_{I_{\alpha}} f_{1}(x) f_{2}\left(T_{\alpha}^{n}(x)\right) d \mu_{\alpha}-\int_{I_{\alpha}} f_{1}(x) d \mu_{\alpha} \int_{I_{\alpha}} f_{2}(x) d \mu_{\alpha}\right| \leq C \lambda^{n}\left\|f_{1}\right\|_{B V}\left\|f_{2}\right\|_{L^{1}} .
$$

Proof. One can take any $\lambda$ such that $\rho\left(\Psi_{\alpha}\right)<\lambda<1$ and $C=2\left\|\rho_{\alpha}\right\|_{B V} \sup _{n \in \mathbb{N}} \frac{\left\|\Psi_{\alpha}^{n}\right\|_{B V}}{\lambda^{n}}$. 


\section{Continuity of entropy}

The goal of this section is to prove Theorem 1.1, namely the Hölder-continuity of the function $\alpha \mapsto h\left(T_{\alpha}\right)$.

The first step is to prove an estimate of the essential spectral radius of the transfer operator acting on the space of BV functions (Lasota-Yorke inequality). If one can prove a bound which is uniform in $\alpha$, then the invariant densities $\rho_{\alpha}$ turn out to be continuous in the $L^{1}$-topology and their $B V$-norms are bounded. This method has been undertaken in [11], but unfortunately their estimates prove to be too optimistic ${ }^{1}$ : the bulk of Section 3.1 (Proposition 3.1) is another proof of this uniform Lasota-Yorke inequality.

The second step (Section 3.2) is to estimate the modulus of continuity of $h\left(T_{\alpha}\right)$ : we will prove Hölder-continuous dependence of the invariant densities $\rho_{\alpha}$ in the $L^{1}$ topology, by using a stability result for the spectral projectors [8]. The theorem then follows from Rohlin's formula.

\subsection{Spectral radius estimate}

We are going to give a proof of the following uniform Lasota-Yorke inequality (in order to simplify notation, from now on $\operatorname{Var}_{I_{\alpha}} f$ will just be denoted $\operatorname{Var} f$ ):

Proposition 3.1. Let $\underline{\alpha} \in(0,1)$. Then there exist a neighbourhood $U$ of $\underline{\alpha}$ and constants $0<\lambda<1, C>0, D>0$ such that for every $\alpha \in U$

$$
\operatorname{Var} \Phi_{\alpha}^{n}(f) \leq C \lambda^{n} \operatorname{Var} f+D\|f\|_{L^{1}} \quad \forall n \geq 1, \forall f \in B V\left(I_{\alpha}\right) .
$$

Although several inequalities of this type are present in the literature, (i.e. in [16]), these are generally given for individual maps. However, for the goal of this paper it is absolutely essential that coefficients $\lambda, C, D$ can be chosen uniformly in $\alpha$, hence one needs to take this dependence into account. As $\alpha$ moves, even just in a neighbourhood of some fixed $\alpha$, topological bifurcations are present (for instance if $\underline{\alpha}$ is a fixed point of some branch of $T_{\underline{\alpha}}$ ) hence in the formula (2.1) new boundary terms appear, requiring a very careful control.

Lemma 3.2. For each $\alpha \in(0,1)$, for each $f \in B V\left(I_{\alpha}\right)$

$$
\operatorname{Var} \Phi_{\alpha}^{n}(f) \leq \operatorname{Var}\left(f \cdot g_{n, \alpha}\right) .
$$

Proof.

$$
\begin{aligned}
\operatorname{Var} \Phi_{\alpha}^{n}(f) & =\operatorname{Var}\left(\sum_{j \in \mathcal{P}_{n}} \frac{f \circ \sigma_{j}}{\left|\left(T_{\alpha}^{n}\right)^{\prime} \circ \sigma_{j}\right|} \chi_{T_{\alpha}^{n}\left(I_{j}\right)}\right) \leq \sum_{j \in \mathcal{P}_{n}} \operatorname{Var}\left(\frac{f \circ \sigma_{j}}{\left|\left(T_{\alpha}^{n}\right)^{\prime} \circ \sigma_{j}\right|} \chi_{T_{\alpha}^{n}\left(I_{j}\right)}\right) \\
& =\sum_{j \in \mathcal{P}_{n}} \operatorname{Var}\left(\frac{f}{\left|\left(T_{\alpha}^{n}\right)^{\prime}\right|} \chi_{I_{j}}\right)=\operatorname{Var}\left(f \sum_{j \in \mathcal{P}_{n}} \frac{1}{\left|\left(T_{\alpha}^{n}\right)^{\prime}\right|} \chi_{I_{j}}\right)=\operatorname{Var}\left(f g_{n, \alpha}\right) .
\end{aligned}
$$

1 The mistake in [11] consists in using, in equation (12), the estimate (1) of Lemma 6.1 of the present paper on the sets $\tilde{I}_{\xi}^{(n)}$, which are not intervals if $n>1$. 
Observe that $g_{n, \alpha}$ has infinitely many jump discontinuities (indeed it is zero on the boundary of any interval of the partition $\mathcal{P}_{n}$ ), but all those jumps sum up to a finite total variation. We will, however, need to prove the stronger statement that the total variation of $g_{n, \alpha}$ decays exponentially fast in $n$, and uniformly in $\alpha$. The idea of the proof is to control the total variation of $g_{n, \alpha}$ by writing it as a sum of two functions, $h_{n, \alpha}$ and $l_{n, \alpha}$ in such a way that the total variation of $l_{n, \alpha}$ is always very small, and $h_{n, \alpha}$ has always a finite, controlled number, of jump discontinuities. The following lemma is the key step:

Lemma 3.3. For each $\epsilon>0$, for each $n \geq 1$, for each $\alpha \in(0,1)$ there exist two non-negative functions $h_{n, \alpha}$ and $l_{n, \alpha}$ such that

$$
g_{n, \alpha}=h_{n, \alpha}+l_{n, \alpha}
$$

and for each $\alpha$ the following holds:

(I) $\operatorname{Var}_{I_{\alpha}} l_{n, \alpha} \leq 3^{n} C_{0}^{n-1} \epsilon$, where $C_{0}$ is the constant in Lemma 2.1;

(II) $h_{n, \alpha}$ is smooth with $\left|h_{n, \alpha}^{\prime}\right| \leq 1$ outside a finite set $J_{n, \alpha}$, where $h_{n, \alpha}$ has jump discontinuities. Moreover, for each $\alpha$ there exists a neighbourhood $U=(\alpha-$ $\eta, \alpha+\eta)$ of $\alpha$ and $r>0$ such that:

(a) For each $\beta \in U, \quad J_{n, \beta} \subseteq B\left(J_{n, \alpha}, r\right)$;

(b) For each $x \in J_{n, \alpha}, \quad \#\left|J_{n, \beta} \cap B(x, r)\right| \leq n+1$;

(c) For each $y \in J_{n, \beta} \cap B(x, r), \quad|x-y| \leq|\alpha-\beta|$.

Proof. By induction on $n$. If $n=1$, let us note that

$$
g_{1, \alpha}(x):= \begin{cases}x^{2} & \text { if } x \text { belongs to some } I_{j} \\ 0 & \text { otherwise }\end{cases}
$$

hence we can choose $L:=\left[-\frac{1}{K}, \frac{1}{K}\right]$ an interval around 0 such that, for all $\alpha$, $\operatorname{Var}_{L} g_{1, \alpha} \leq \epsilon$ and define

$$
l_{1, \alpha}:=g_{1, \alpha} \chi_{L} \quad h_{1, \alpha}:=g_{1, \alpha} \chi_{I_{\alpha} \backslash L} .
$$

(I) is clearly verified. To verify (II), note that given $x \in J_{1, \alpha}, x \neq \alpha, \alpha-1$, for $\beta$ sufficiently close to $\alpha, J_{1, \beta}$ intersects a neighbourhood of $x$ in only one point. The same happens if $x=\alpha, \alpha-1$ and $T_{\alpha}(x) \neq \alpha-1$. On the other hand, if $x=\alpha$ and $T_{\alpha}(\alpha)=\alpha-1$, then $J_{1, \beta} \cap[\beta-\eta, \beta]=\{y, \beta\}$ contains at most two points, where $y=T_{\beta}^{-1}(\beta-1) \cap[\beta-\eta, \beta]$ and, since $T_{\beta}$ is expanding, $|y-\alpha| \leq|\alpha-\beta|$. The case $x=\alpha-1, T_{\alpha}(\alpha-1)=\alpha-1$ is similar.

In order to prove the inductive step, let us remark that $g_{n+1, \alpha}=g_{n, \alpha} \circ T_{\alpha} \cdot g_{1, \alpha}$. Hence, we can define

$$
\begin{aligned}
& h_{n+1, \alpha}:=h_{n, \alpha} \circ T_{\alpha} \cdot h_{1, \alpha} \\
& l_{n+1, \alpha}:=l_{n, \alpha} \circ T_{\alpha} \cdot g_{1, \alpha}+h_{n, \alpha} \circ T_{\alpha} \cdot l_{1, \alpha}
\end{aligned}
$$


and check that all the properties are satisfied. First of all, we can prove by induction that

$$
\operatorname{Var}_{I_{\alpha}} h_{n, \alpha} \leq 2^{n-1} C_{0}^{n} \quad \forall \alpha \in(0,1), \forall n \geq 1 \text {. }
$$

Indeed,

$$
\begin{aligned}
\operatorname{Var}_{I_{\alpha}} h_{1, \alpha} & \leq \operatorname{Var}_{I_{\alpha}} g_{1, \alpha} \leq C_{0} \operatorname{Var}_{I_{\alpha}} h_{n+1, \alpha}=\sum_{k \in \mathcal{P}_{1}} \operatorname{Var}_{\bar{I}_{k}}\left(h_{n, \alpha} \circ T_{\alpha} \cdot h_{1, \alpha}\right) \\
& \leq \sum_{k \in \mathcal{P}_{1}} \operatorname{Var}_{\bar{I}_{k}}\left(h_{n, \alpha} \circ T_{\alpha}\right) \sup _{\bar{I}_{k}} h_{1, \alpha}+\sup _{\bar{I}_{k}}\left(h_{n, \alpha} \circ T_{\alpha}\right) \operatorname{Var}_{\bar{I}_{k}} h_{1, \alpha}
\end{aligned}
$$

and since $\left.T_{\alpha}\right|_{I_{k}}$ is a homeomorphism

$$
\begin{aligned}
& \leq \operatorname{Var}_{I_{\alpha}} h_{n, \alpha} \sum_{k \in \mathcal{P}_{1}} \sup _{\bar{I}_{k}} h_{1, \alpha}+\sup _{I_{\alpha}} h_{n, \alpha} \sum_{k \in \mathcal{P}_{1}} \operatorname{Var}_{\bar{I}_{k}} h_{1, \alpha} \\
& \leq 2 \operatorname{Var}_{I_{\alpha}} h_{n, \alpha} \operatorname{Var}_{I_{\alpha}} h_{1, \alpha} \leq 2 \cdot 2^{n-1} C_{0}^{n} \cdot C_{0}
\end{aligned}
$$

where in the penultimate inequality we used the fact that $\sup _{I} f \leq \operatorname{Var}_{I} f$ if $f(x)=$ 0 for some $x \in I$.

Let us now check (I): similarly as before,

$$
\begin{aligned}
\operatorname{Var}_{I_{\alpha}} l_{n+1, \alpha} & =\operatorname{Var}_{I_{\alpha}}\left(l_{n, \alpha} \circ T_{\alpha} \cdot g_{1, \alpha}+h_{n, \alpha} \circ T_{\alpha} \cdot l_{1, \alpha}\right) \\
& \leq 2 \operatorname{Var}_{I_{\alpha}} l_{n, \alpha} \operatorname{Var}_{I_{\alpha}} g_{1, \alpha}+2 \operatorname{Var}_{I_{\alpha}} h_{n, \alpha} \operatorname{Var}_{I_{\alpha}} l_{1, \alpha}
\end{aligned}
$$

and by inductive hypothesis and (3.1)

$$
\leq 2 \cdot 3^{n} C_{0}^{n-1} \epsilon \cdot C_{0}+2 \cdot 2^{n-1} C_{0}^{n} \cdot \epsilon \leq 3^{n+1} C_{0}^{n} \epsilon .
$$

Since $h_{1, \alpha}$ is nonzero only on finitely many branches of $T_{\alpha}$, then $h_{n+1, \alpha}$ has only finitely many jump discontinuities. Now, if $x$ is a jump discontinuity for $h_{n, \alpha} \circ T_{\alpha}$ and not for $h_{1, \alpha}$, then $T_{\beta}$ is an expanding local homeomorphism at $x$ for all $\beta$ in a neighbourhood of $\alpha$, hence (a), (b) and (c) follow. Let now $x \neq \alpha, \alpha-1$ be on the boundary of some cylinder, i.e. $T_{\alpha}(x)=\alpha-1$. Then by inductive hypothesis (c), if $\beta>\alpha$ is sufficiently close to $\alpha$ and $\eta$ is sufficiently small, then

$$
J_{n, \beta} \cap[\beta-1, \beta-1+\eta]=\{\beta-1\}
$$

hence

$$
J_{n+1, \beta} \cap B(x, r)=T_{\beta}^{-1}\left(J_{n, \beta} \cap[\beta-\eta, \beta]\right) \cap B(x, r)
$$

and (b) follows. (c) follows from the fact that $T_{\beta}$ is expanding. If $\beta<\alpha$, similarly the claims follow because

$$
J_{n+1, \beta} \cap B(x, r)=T_{\beta}^{-1}\left(J_{n, \beta} \cap[\beta-1, \beta-1+\eta]\right) \cap B(x, r) .
$$

If $x=\alpha$, then for $\beta$ sufficiently close to $\alpha$,

$$
J_{n+1, \beta} \cap B(x, r) \subseteq\left(T_{\beta}^{-1}\left(J_{n, \beta}\right) \cup\{\beta\}\right) \cap B(x, r)
$$

has cardinality at most $n+2$, and (c) follows because $T_{\alpha}$ is expanding. The case $x=\alpha-1$ is analogous. 
Lemma 3.4. Let $\alpha \in(0,1), n \geq 1$ and $\epsilon>0$. Then there exist $\eta>0, C>0$ and a finite partition of $[\alpha-1-\eta, \alpha+\eta]$ in closed intervals $L_{1}, \ldots L_{r}$ such that for each $\beta \in(\alpha-\eta, \alpha+\eta)$ and each $i \in 1, \ldots, r$ the following holds:

- $0<C \leq m\left(L_{i, \beta}\right) \leq \epsilon$

- $\operatorname{Var}_{L_{i, \beta}} g_{n, \beta} \leq 2(n+1)\left\|g_{n, \beta}\right\|_{\infty}+2 \epsilon$

where $L_{i, \beta}:=L_{i} \cap[\beta-1, \beta]$.

Proof. Given $\alpha, n, \epsilon$, choose $L_{1}, \ldots, L_{r}$ in such a way that $m\left(L_{i}\right) \leq \epsilon$, each element of $J_{n, \alpha}$ lies in the interior of some $L_{i}$ and no two such elements lie in the same $L_{i}$. Moreover, set $\epsilon_{1}:=\epsilon /\left(3^{n} C_{0}^{n-1}\right)$ and, for each $\beta$ sufficiently close to $\alpha$, choose a decomposition $g_{n, \beta}=h_{n, \beta}+l_{n, \beta}$ as in Lemma 3.3 relative to $\epsilon_{1}$.

$$
\begin{aligned}
\operatorname{Var}_{L_{i, \beta}} h_{n, \beta} & \leq \int_{L_{i, \beta} \backslash J_{n, \beta}} h_{n, \beta}^{\prime}(x) d x+\sum_{x \in L_{i, \beta} \cap J_{n, \beta}} \lim _{y \rightarrow x^{-}} h_{n, \beta}(y)+\lim _{y \rightarrow x^{+}} h_{n, \beta}(y) \\
& \leq m\left(L_{i, \beta}\right)+2 \#\left\{L_{i, \beta} \cap J_{n, \beta}\right\}\left\|h_{n, \beta}\right\|_{\infty} \leq \epsilon+2(n+1)\left\|h_{n, \beta}\right\|_{\infty}
\end{aligned}
$$

hence $\operatorname{Var}_{L_{i, \beta}} g_{n, \beta} \leq \operatorname{Var}_{L_{i, \beta}} h_{n, \beta}+l_{n, \beta} \leq 2 \epsilon+2(n+1)\left\|g_{n, \beta}\right\|_{\infty}$.

Proof of Proposition 3.1. Consider the partition $L_{1}, \ldots, L_{r}$ given by Lemma 3.4. Then

$$
\begin{aligned}
\operatorname{Var}\left(f \cdot g_{n, \alpha}\right) & =\sum_{i=1}^{r} \operatorname{Var}_{L_{i}}\left(f g_{n, \alpha}\right) \leq \sum_{i=1}^{r} \operatorname{Var}_{L_{i}} f \sup _{L_{i}} g_{n, \alpha}+\operatorname{Var}_{L_{i}} g_{n, \alpha} \sup _{L_{i}} f \\
& \leq \sum_{i=1}^{r}\left\|g_{n, \alpha}\right\|_{\infty} \operatorname{Var}_{L_{i}} f+\operatorname{Var}_{L_{i}} g_{n, \alpha}\left(\frac{1}{m\left(L_{i, \alpha}\right)} \int_{L_{i}} f(x) d x+\operatorname{Var}_{L_{i}} f\right) \\
& \leq\left[(2 n+3)\left\|g_{n, \alpha}\right\|_{\infty}+2 \epsilon\right] \operatorname{Var}_{I_{\alpha}} f+\frac{(2 n+2)\left\|g_{n, \alpha}\right\|_{\infty}+2 \epsilon}{C} \int_{I_{\alpha}} f(x) d x .
\end{aligned}
$$

Now, since $\left\|g_{n, \alpha}\right\|_{\infty} \leq \gamma_{\alpha}^{n}$ decays exponentially, we can choose $n$ large enough so that $\lambda:=(2 n+4) \gamma_{\alpha}^{n}<1$, and we can also choose $2 \epsilon \leq \gamma_{\alpha}^{n}$, hence we get that for some constant $D>0$, for each $\alpha \in(\underline{\alpha}-\eta, \underline{\alpha}+\eta)$,

$$
\operatorname{Var} \Phi_{\alpha}^{n}(f) \leq \lambda \operatorname{Var} f+D\|f\|_{1}
$$

and by iteration and euclidean division (see e.g. [16, Lemma 7 and Proposition 1] the claim is proven.

\subsection{Stability of the spectral decomposition}

The next step to prove Hölder-continuity is proving the continuous dependence of the invariant densities $\rho_{\alpha}$ in $L^{1}$-norm. In order to guarantee the stability of spectral projectors of the transfer operator, we will use the following theorem of Keller and Liverani [8]: 
Theorem 3.5. Let $P_{\epsilon}$ be a family of bounded linear operators on a Banach space $(B,\|\cdot\|)$ which is also equipped with a second norm $|\cdot|$ such that $|\cdot| \leq\|\cdot\|$. Let us assume that the following conditions hold:

(1) $\exists C_{1}, M>0$ such that for all $\epsilon \geq 0$

$$
\left|P_{\epsilon}^{n}\right| \leq C_{1} M^{n} \quad \forall n \in \mathbb{N}
$$

(2) $\exists C_{2}, C_{3}>0$ and $\lambda \in(0,1), \lambda<M$, such that for all $\epsilon \geq 0$

$$
\left\|P_{\epsilon}^{n} f\right\| \leq C_{2} \lambda^{n}\|f\|+C_{3} M^{n}|f| \quad \forall n \in \mathbb{N} \quad \forall f \in B ;
$$

(3) if $z \in \sigma\left(P_{\epsilon}\right),|z|>\lambda$, then $z$ is not in the residual spectrum of $P_{\epsilon}$;

(4) there is a monotone continuous function $\tau:[0, \infty) \rightarrow[0, \infty)$ such that $\tau(\epsilon)>0$ if $\epsilon>0$ and

$$
\left\|P_{0}-P_{\epsilon}\right\| \leq \tau(\epsilon) \rightarrow 0 \quad \text { as } \epsilon \rightarrow 0
$$

where the norm $\|\cdot\|$ is defined as

$$
\|Q\|:=\sup _{\|f\| \leq 1}|Q f|
$$

Let us now fix $\delta>0$ and $r \in(\lambda, M)$ and define

$$
V_{\delta, r}:=\left\{z \in \mathbb{C}:|z| \leq r \text { or dist }\left(z, \sigma\left(P_{0}\right)\right) \leq \delta\right\}
$$

and $\eta:=\frac{\log (\lambda / r)}{\log (\lambda / M)}$. Then there exist $H, K>0$ such that if $\tau(\epsilon) \leq H$ then $\sigma\left(P_{\epsilon}\right) \subseteq$ $V_{\delta, r}$ and

$$
\left\|\left(z-P_{\epsilon}\right)^{-1}-\left(z-P_{0}\right)^{-1}\right\| \leq K \tau(\epsilon)^{\eta} \quad \forall z \notin V_{\delta, r} .
$$

In our context, the norm $|\cdot|$ will be the $L^{1}$ norm and $\|\cdot\|$ will be the $B V$ norm. Our goal is to apply this result to the family $\left\{\Phi_{\alpha}\right\}_{\alpha \in U}$ where $U$ is a suitable neighbourhood of a given $\underline{\alpha} \in(0,1)$.

Hypothesis (1) is trivial since transfer operators have unit $L^{1}$-norm, and (2) is precisely Proposition 3.1. In the context of one-dimensional piecewise expanding maps, (3) is an immediate corollary of (2):

Lemma 3.6. For every $\underline{\alpha} \in(0,1)$ there exists $\epsilon>0$ such that for $|\alpha-\underline{\alpha}|<\epsilon$,

$$
\rho_{\text {ess }}\left(\Phi_{\alpha}\right) \leq \lambda
$$

where $\lambda$ is the same as in Proposition 3.1 and therefore condition (3) holds.

Proof. By a result of Hennion [5], the uniform Lasota-Yorke inequality plus the fact that the injection $B V(I) \rightarrow L^{1}(I)$ is compact implies the estimate on the essential spectral radius; therefore the elements of the spectrum of modulus bigger than $\lambda$ are eigenvalues with finite multiplicity and cannot belong to the residual spectrum. 
To prove condition (4) it is necessary to estimate the distance between the $\Phi_{\alpha}$ as $\alpha$ varies in a neighbourhood of a fixed $\alpha$; by a result of Keller [7] the distance between the transfer operators is related to the following distance between the transformations:

Definition 3.7. Let $T_{1}, T_{2}: I \rightarrow I$ two maps of the interval $I$. We define the Keller distance between $T_{1}$ and $T_{2}$ as

$$
\begin{gathered}
d\left(T_{1}, T_{2}\right):=\inf \{\kappa>0 \mid \exists A \subset I \text { measurable with } m(A)>1-\kappa, \\
\exists \sigma: I \rightarrow I \text { diffeomorphism such that }\left.T_{1}\right|_{A}=\left.T_{2} \circ \sigma\right|_{A}, \\
\left.\sup _{x \in I}|\sigma(x)-x|<\kappa, \sup _{x \in I}\left|\frac{1}{\sigma^{\prime}(x)}-1\right|<\kappa\right\} .
\end{gathered}
$$

Lemma 3.8 ([7, Lemma 13]). If $P_{1}$ and $P_{2}$ are the transfer operators associated to the interval maps $T_{1}$ and $T_{2}$, then $\left\|P_{1}-P_{2}\right\| \leq 12 d\left(T_{1}, T_{2}\right)$ where $d$ is the Keller distance.

We verify now that this convergence result applies to our case of $\alpha$-continued fractions. In order to do so, it is necessary to translate the maps in such a way that they are all defined on the same interval, which will be $[0,1]$ in our case. We therefore consider the maps $\tilde{T}_{\alpha}:[0,1] \rightarrow[0,1]$

$$
\tilde{T}_{\alpha}(x)=T_{\alpha}(x+\alpha-1)+1-\alpha .
$$

The relative invariant densities will be

$$
\tilde{\rho}_{\alpha}(x)=\rho_{\alpha}(x+\alpha-1) .
$$

Lemma 3.9. Fix $\underline{\alpha} \in(0,1)$. Then there exists a neighbourhood $U$ of $\underline{\alpha}$ and a positive constant $\bar{C}$ such that, for $\alpha, \beta \in U$, we have

$$
d\left(\tilde{T}_{\alpha}, \tilde{T}_{\beta}\right) \leq C|\alpha-\beta|^{1 / 2} .
$$

Proof. Having fixed $\alpha, \beta$, let us define

$$
y(x):=\frac{x+\alpha-1}{1+(\beta-\alpha)|x+\alpha-1|}+1-\beta .
$$

It is immediate to verify that $\tilde{T}_{\alpha}(x)=\tilde{T}_{\beta}(y(x)) \forall x \in[0,1]$ and $y^{\prime}(x)=$ $\frac{1}{(1+(\beta-\alpha)|x+\alpha-1|)^{2}}$. We also have

$$
\sup _{x \in[0,1]}|y(x)-x|=|y(1)-1|
$$


because when $|\alpha-\beta|$ is small we have that, for $\alpha>\beta, y(x)-x$ has positive derivative and $y(0)>0$, while, for $\alpha<\beta, y(x)-x$ has negative derivative and $y(0)<0$. Thus, for $|\alpha-\beta|$ sufficiently small,

$$
\begin{aligned}
& \sup _{x \in[0,1]}|y(x)-x|=|\alpha-\beta|\left|\frac{1+\alpha \beta}{1+(\beta-\alpha) \alpha}\right| \leq 2|\alpha-\beta| \\
& \sup _{x \in[0,1]}\left|\frac{1}{y^{\prime}(x)}-1\right|=|\beta-\alpha| \sup _{x \in[0,1]}|2| x+\alpha-1|+(\beta-\alpha)| x+\alpha-\left.1\right|^{2}|\leq 3| \alpha-\beta \mid .
\end{aligned}
$$

In order to compute the Keller distance we need to find a diffeomorphism $\sigma$ of the interval such that $\tilde{T}_{\alpha}=\tilde{T}_{\beta} \circ \sigma$ on a set of large measure; the $y$ defined so far is not a diffeomorphism, so it is necessary to modify it a bit at the endpoints and we will do it by introducing two little linear bridges. Let $\delta$ be such that $\delta^{2}=$ $\sup _{x \in[0,1]}|y(x)-x| \leq 2|\alpha-\beta|$; we can define

$$
\sigma(x)= \begin{cases}\frac{y(\delta)}{\delta} x & \text { for } x \leq \delta \\ y(x) & \text { for } \delta \leq x \leq 1-\delta \\ \frac{1-y(1-\delta)}{\delta}(x-1+\delta)+y(1-\delta) & \text { for } x \geq 1-\delta .\end{cases}
$$

For the sup norm we have

$$
\begin{aligned}
\sup _{x \in[0,1]}|\sigma(x)-x| & \leq \max \left\{|y(\delta)-\delta|, \sup _{x \in[\delta, 1-\delta]}|y(x)-x|,|y(1-\delta)-1+\delta|\right\} \\
& \leq \sup _{x \in[0,1]}|y(x)-x| \leq 2|\beta-\alpha| .
\end{aligned}
$$

Since $|y(\delta)| \geq \delta-|y(\delta)-\delta| \geq \delta-\delta^{2}$, one gets $\sup _{x \in[0, \delta]}\left|\frac{1}{\sigma^{\prime}(x)}-1\right| \leq \frac{\delta}{1-\delta}$ and

$$
\sup _{x \in[0,1]}\left|\frac{1}{\sigma^{\prime}(x)}-1\right| \leq \max \left\{3|\alpha-\beta|, \frac{\delta}{1-\delta}\right\} \leq C|\alpha-\beta|^{1 / 2} .
$$

Now, $\sigma$ is a homeomorphism of $[0,1]$ with well-defined, non-zero derivative except for the points $x=\delta, 1-\delta$. Hence one can construct smooth approximations $\sigma_{n}$ of $\sigma$ which coincide with it except on $\left[\delta-\frac{1}{2^{n}}, \delta+\frac{1}{2^{n}}\right] \cup\left[1-\delta-\frac{1}{2^{n}}, 1-\delta+\frac{1}{2^{n}}\right]$ and such that the previous estimates still hold. These $\sigma_{n}$ will be diffeomorphisms of the interval such that $\tilde{T}_{\alpha}(x)=\tilde{T}_{\beta}\left(\sigma_{n}(x)\right)$ for $x \in\left[\delta+\frac{1}{2^{n}}, 1-\delta-\frac{1}{2^{n}}\right]$. Since $\sup m\left(\left[\delta+\frac{1}{2^{n}}, 1-\delta-\frac{1}{2^{n}}\right]\right)=1-2 \delta \geq 1-2|\alpha-\beta|^{1 / 2}$, the claim is proven.

\subsection{Hölder-continuity of the entropy}

By using the perturbation theory developed so far, we complete the proof that the function $\alpha \mapsto h\left(T_{\alpha}\right)$ is locally Hölder-continuous. Note that the uniform LasotaYorke inequality proven in Section 3.1 would already imply continuity by the methods in [11], while here we get a quantitative bound on the continuity module. 
Proposition 3.10. Let $\delta>0$, and $0<s<\frac{1}{2}$. Then there exists a constant $C>0$ such that

$$
\left|h\left(T_{\alpha}\right)-h\left(T_{\beta}\right)\right| \leq C|\alpha-\beta|^{s} \quad \forall \alpha, \beta \in[\delta, 1] .
$$

Proof. Let us fix $\eta \in(0,1)$, and choose $r$ such that $\eta=\frac{\log (\lambda / r)}{\log (\lambda)}$. By Theorem 3.5 applied to the family $\Phi_{\alpha}$, (using Proposition 3.1, Lemma 3.6 and Lemma 3.9 as hypotheses), for each $\alpha \in(0,1)$ there exist $\epsilon, C_{1}>0$ such that

$$
\left\|\Pi_{\alpha}-\Pi_{\beta}\right\| \leq C_{1}|\alpha-\beta|^{\eta / 2} \quad \forall \beta \in(\alpha-\epsilon, \alpha+\epsilon) .
$$

Now, in Theorem 3.5 the bounds $(H, K)$ depend only on the constants $C_{1}, C_{2}, C_{3}$, $\lambda, M$, and in Proposition 3.1 and Lemma 3.9 these constants are locally uniformly bounded in $\alpha$, hence the following stronger statement is true: for each $\underline{\alpha} \in(0,1)$ there is $C_{1}>0$ and some neighbourhood $U$ of $\underline{\alpha}$ such that

$$
\left\|\Pi_{\alpha}-\Pi_{\beta}\right\| \leq C_{1}|\alpha-\beta|^{\eta / 2} \quad \forall \alpha, \beta \in U
$$

Since $\tilde{\rho_{\alpha}}=\Pi_{\alpha}(1)$, the previous equation implies

$$
\left\|\tilde{\rho}_{\alpha}-\tilde{\rho}_{\beta}\right\|_{L^{1}}=O\left(|\alpha-\beta|^{\frac{\eta}{2}}\right) .
$$

By Proposition 3.1, $\left\|\tilde{\rho}_{\alpha}\right\|_{B V}$ is locally bounded, hence so is $\left\|\tilde{\rho}_{\alpha}\right\|_{\infty}$ and for any $p>1$

$$
\left\|\tilde{\rho}_{\alpha}-\tilde{\rho}_{\beta}\right\|_{L^{p}}=O\left(|\alpha-\beta|^{\frac{\eta}{2 p}}\right) .
$$

By Rohlin's formula, $h\left(T_{\alpha}\right)=-2 \int_{0}^{1} \log |y+\alpha-1| \tilde{\rho}_{\alpha}(y) d y$, thus

$$
\left|h\left(T_{\alpha}\right)-h\left(T_{\beta}\right)\right| \leq 2 \int_{0}^{1}|\log | y+\alpha-1\left|\tilde{\rho}_{\alpha}(y)-\log \right| y+\beta-1\left|\tilde{\rho}_{\beta}(y)\right| d y
$$

by separating the product and applying Hölder's inequality, for any $p>1$

$\leq 2\left\|\tilde{\rho}_{\alpha}\right\|_{\infty}\|\log |y+\alpha-1|-\log |y+\beta-1|\|_{L^{1}}+\|2 \log (y+\beta-1)\|_{L^{p / p-1}}\left\|\tilde{\rho}_{\alpha}-\tilde{\rho}_{\beta}\right\|_{L^{p}}$.

Now, basic calculus shows $\|\log |y+\alpha-1|-\log |y+\beta-1|\|_{L^{1}}=O(-\mid \alpha-$ $\beta|\log | \alpha-\beta \mid)$ and $\|2 \log (y+\alpha-1)\|_{L^{p / p-1}}$ is bounded independently of $\alpha$. Since this is true $\forall \eta<1$ and $\forall p>1$, the claim follows.

Remark 3.11. One has to be careful with the norm one uses to get the convergence, because while $L^{1}$-convergence of the densities is assured by uniform Lasota-Yorke, the invariant densities in general DO NOT converge to each other in $B V$-norm. For example we have for $\alpha \geq \frac{\sqrt{5}-1}{2}$

$$
\rho_{\alpha}(x)=\frac{1}{\log (1+\alpha)}\left(\chi_{\left[0, \frac{1-\alpha^{2}}{\alpha}\right]}(x) \frac{1}{x+2}+\chi_{\left(\frac{1-\alpha^{2}}{\alpha}, 1\right]}(x) \frac{1}{x+1}\right)
$$


SO

$$
\operatorname{Var}_{[0,1]}\left(\tilde{\rho}_{\alpha}-\tilde{\rho}_{\underline{\alpha}}\right) \geq\left|\lim _{x \rightarrow\left(\frac{1-\alpha^{2}}{\alpha}\right)^{-}}\left(\rho_{\alpha}-\rho_{\underline{\alpha}}\right)-\lim _{x \rightarrow\left(\frac{1-\alpha^{2}}{\alpha}\right)^{+}}\left(\rho_{\alpha}-\rho_{\underline{\alpha}}\right)\right|
$$

which does not converge to 0 as $\alpha \rightarrow \underline{\alpha}$.

Finally, let us remark why we think the entropy is not locally Lipschitz. Indeed, there is a formula to compute the difference quotient of $h\left(T_{\alpha}\right)$ on monotonicity intervals. More precisely, every maximal quadratic interval $I_{r}$ of [3] is parametrized by a rational number $r$, and the monotonic behaviour of $h\left(T_{\alpha}\right)$ on $I_{r}$ is controlled by the usual continued fraction expansion of $r$. Indeed, if $r=\left[0 ; a_{1}, \ldots, a_{n}\right]$ is the continued fraction expansion of $r$ with $n$ even, let us call matching index the quantity $\llbracket r \rrbracket:=\sum_{i=1}^{n}(-1)^{i+1} a_{i}$ : the formula of [3, Lemma A.4] (already present in [14, proof of Theorem 2]) tells you that

$$
\frac{h\left(T_{\alpha^{\prime}}\right)-h\left(T_{\alpha}\right)}{\alpha^{\prime}-\alpha}=h\left(T_{\alpha}\right) \llbracket r \rrbracket \frac{\mu_{\alpha^{\prime}}\left(\left[\alpha, \alpha^{\prime}\right]\right)}{\alpha^{\prime}-\alpha}
$$

whenever $\alpha$ and $\alpha^{\prime}$ belong to $I_{r}$ and are sufficiently close to each other. Now, it is not hard to find a sequence of maximal quadratic intervals $I_{r_{n}}$ which accumulate on some parameter $\tilde{\alpha}$ and such that $\llbracket r_{n} \rrbracket \rightarrow \infty$ (a way to produce such a family is to iterate the tuning construction of [4]). Let us pick a sequence $\left(\alpha_{n}, \alpha_{n}^{\prime}\right)$ of pairs of parameters such that $\alpha_{n}, \alpha_{n}^{\prime}$ belong to $I_{r_{n}}$ and equation (3.3) holds.

Numerical evidence suggests that the invariant density $\rho_{\alpha}$ is locally uniformly bounded below by a positive constant, which would imply $\inf _{\substack{\alpha \rightarrow \tilde{\alpha} \\ \alpha^{\prime} \rightarrow \tilde{\alpha}}} \frac{\mu_{\alpha^{\prime}}\left(\alpha, \alpha^{\prime}\right)}{\left|\alpha^{\prime}-\alpha\right|}>0$, hence

$$
\lim _{n \rightarrow \infty}\left|\frac{h\left(T_{\alpha_{n}^{\prime}}\right)-h\left(T_{\alpha_{n}}\right)}{\alpha_{n}^{\prime}-\alpha_{n}}\right|=+\infty .
$$

\section{Central limit theorems}

The goal of this section is to prove a central limit theorem (CLT) for the systems $T_{\alpha}$. Given an observable $f: I_{\alpha} \rightarrow \mathbb{R}$, we denote by $S_{n} f$ the Birkhoff sum

$$
S_{n} f=\sum_{j=0}^{n-1} f \circ T_{\alpha}^{j} .
$$

The function $x \mapsto \frac{S_{n} f(x)}{n}$ is called Birkhoff average and it can be seen as a random variable on the space $I_{\alpha}=[\alpha-1, \alpha]$ endowed with the measure $\mu_{\alpha}$. By ergodicity, this random variable converges a.e. to a constant. Our goal is to prove that the difference from such limit value converges in law to a Gaussian distribution.

Heuristically, this means the sequence of observables $\left\{f \circ T_{\alpha}^{n}\right\}$ (which can be seen as identically distributed random variables on $I_{\alpha}$ ) behave as if they were 
independent, i.e. the system has little memory of its past. A convergence property of this type is also useful to confirm numerical data, since it implies the variance of Birkhoff averages up to the $n^{\text {th }}$ iterate decays as $\frac{1}{\sqrt{n}}$, hence one can get a good approximation of the limit value by computing Birkhoff averages up to a suitable finite time $n$ (see [11]).

First (Subsection 4.1), we will prove CLT for observables of bounded variation. A particularly important observable is $\log \left|T_{\alpha}^{\prime}\right|$, because by Rohlin's formula its expectation is the metric entropy. Such observable, however, is not of bounded variation: in Subsection 4.2, we will enlarge the class of observables we work with in order to encompass certain unbounded functions, including $\log \left|T_{\alpha}^{\prime}\right|$. In order to do so, we need to define ad hoc Banach spaces.

\subsection{CLT for functions of bounded variation}

Theorem 4.1. Let $\alpha \in(0,1]$ and $f$ be a real-valued nonconstant element of $B V\left(I_{\alpha}\right)$. There exists $\sigma>0$ such that the random variable $\frac{S_{n}\left(f-\int f d \mu_{\alpha}\right)}{\sigma \sqrt{n}}$ converges in law to a Gaussian $\mathcal{N}(0,1)$, i.e. for every $v \in \mathbb{R}$ we have

$$
\lim _{n \rightarrow \infty} \mu_{\alpha}\left(\frac{S_{n} f-n \int_{I} f d \mu_{\alpha}}{\sigma \sqrt{n}} \leq v\right)=\frac{1}{\sqrt{2 \pi}} \int_{-\infty}^{v} e^{-x^{2} / 2} d x .
$$

The proof of Theorem 4.1 follows a method developed by A. Broise [1].

\section{Perturbations of $\Phi_{\alpha}$}

Given $f \in B V\left(I_{\alpha}\right)$ with real values and given $\theta \in \mathbb{C}$, let us define the operator $\Phi_{f}(\theta): B V\left(I_{\alpha}\right) \rightarrow B V\left(I_{\alpha}\right)$ with

$$
\Phi_{f}(\theta)(g)=\Phi(\exp (\theta f) g) .
$$

For fixed $f$, this family of operators has the property that $\Phi_{f}(0)=\Phi$ and the function $\theta \mapsto \Phi_{f}(\theta)$ is analytic; the interest in this kind of perturbations resides in the identity

$$
\Phi_{f}^{n}(\theta)(g)=\Phi^{n}\left(\exp \left(\theta S_{n} f\right) g\right) \quad \text { with } S_{n} f=\sum_{k=0}^{n-1} f \circ T_{\alpha}^{k} .
$$

Since in our case all the eigenvalues of modulus 1 are simple, the spectral decomposition transfers to the perturbed operator:

$$
\Phi_{f}^{n}(\theta)(g)=\lambda_{0}^{n}(\theta) \Phi_{0}(\theta)(g)+\Psi_{f}^{n}(\theta)(g)
$$

where the functions $\theta \mapsto \Phi_{0}(\theta), \theta \mapsto \lambda_{0}(\theta)$ and $\theta \mapsto \Psi_{f}(\theta)$ are analytic in a neighbourhood of $\theta=0$. Moreover, $\rho\left(\Psi_{f}(\theta)\right) \leq \frac{2+\rho(\Psi)}{3} \leq\left|\lambda_{0}(\theta)\right|$.

Let us now consider the variance of $S_{n} f$ : 
Proposition 4.2 ([1, Theorem 6.1]). Let $\alpha \in(0,1]$ and $f$ be a real-valued element of $B V\left(I_{\alpha}\right)$. Then the sequence

$$
M_{n}=\int_{I_{\alpha}}\left(\frac{S_{n} f-n \int f d \mu_{\alpha}}{\sqrt{n}}\right)^{2} d \mu_{\alpha}
$$

converges to a real non-negative value, which will be denoted by $\sigma^{2}$. Moreover, $\sigma^{2}=0$ if and only if there exists $u \in L^{2}\left(\mu_{\alpha}\right)$ such that $u \rho_{\alpha} \in B V\left(I_{\alpha}\right)$ and

$$
f-\int_{I_{\alpha}} f d \mu_{\alpha}=u-u \circ T_{\alpha} .
$$

Now, if $\sigma>0$, the method of [1, Chapter 6] yields the central limit theorem. The main steps in the argument are:

(1) $\lambda_{0}^{\prime}(0)=\int_{I_{\alpha}} f d \mu_{\alpha}$;

(2) If $\int_{I_{\alpha}} f d \mu_{\alpha}=0$, then $\lambda_{0}^{\prime \prime}(0)=\sigma^{2}$;

(3) If $\int_{I_{\alpha}} f d \mu_{\alpha}=0$, then $\lim _{n \rightarrow \infty} \int_{I_{\alpha}} \Phi_{f}^{n}\left(\frac{i t}{\sigma \sqrt{n}}\right)\left(\rho_{\alpha}\right) d m=\exp \left(-\frac{t^{2}}{2}\right)$.

The CLT then follows by Lévy's continuity theorem, the left-hand side of previous equation being the characteristic function of the random variable $\frac{S_{n}\left(f-\int_{I_{\alpha}} f d \mu_{\alpha}\right)}{\sigma \sqrt{n}}$.

In order to prove the CLT for a given observable we are now left with checking that Equation (4.1) has no solutions. The following proposition completes the proof of Theorem 4.1 .

Proposition 4.3. For every real-valued nonconstant $f \in B V\left(I_{\alpha}\right)$, Equation (4.1) has no solutions.

Proof. By Proposition 2.1, $T_{\alpha}$ satisfies the hypotheses of a theorem of Zweimüller [19], which asserts that there exists $C_{\alpha}>0$ such that $\rho_{\alpha} \geq C_{\alpha}$ on $\left\{\rho_{\alpha} \neq 0\right\}$. Hence, the function $\frac{1}{\rho_{\alpha}} \chi_{\left\{\rho_{\alpha} \neq 0\right\}}$ belongs to $B V\left(I_{\alpha}\right)$, so if it exists $u$ such that $f \rho_{\alpha}-$ $\left(\int_{I_{\alpha}} f d \mu_{\alpha}\right) \rho_{\alpha}=u \rho_{\alpha}-u \circ T_{\alpha} \cdot \rho_{\alpha}$ in $B V\left(I_{\alpha}\right)$, then we can multiply by $\frac{1}{\rho_{\alpha}} \chi_{\left\{\rho_{\alpha} \neq 0\right\}}$ and get $f-\int_{I_{\alpha}} f d \mu_{\alpha}=u-u \circ T_{\alpha}$ in $B V\left(I_{\alpha}\right)$, with $u$ in $B V\left(I_{\alpha}\right)$ because $u \rho_{\alpha} \in B V\left(I_{\alpha}\right)$; by knowing that $f \in B V\left(I_{\alpha}\right)$ we get $u \circ T_{\alpha} \in B V\left(I_{\alpha}\right)$. For each cylinder $I_{j} \in \mathcal{P}_{1}$, since $\left.T_{\alpha}\right|_{I_{j}}: I_{j} \rightarrow I_{\alpha}$ is a homeomorphism,

$$
\operatorname{Var}_{I_{j}}\left(u \circ T_{\alpha}\right)=\operatorname{Var}_{T_{\alpha}\left(I_{j}\right)} u
$$

hence

$$
\operatorname{Var}_{I_{\alpha}}\left(u \circ T_{\alpha}\right) \geq \sum_{\substack{I_{j} \in \mathcal{P}_{1} \\ I_{j} \text { full }}} \operatorname{Var}_{I_{j}}\left(u \circ T_{\alpha}\right) \geq \sum_{\substack{I_{j} \in \mathcal{P}_{1} \\ I_{j} \text { full }}} \operatorname{Var}_{T_{\alpha}\left(I_{j}\right)} u=\sum_{\substack{I_{j} \in \mathcal{P}_{1} \\ I_{j} \text { full }}} \operatorname{Var}_{(\alpha-1, \alpha)} u
$$

and, since the set of $j$ such that $I_{j}$ is full is infinite, $u \circ T_{\alpha}$ has a representative with bounded variation only if $\operatorname{Var}_{(\alpha-1, \alpha)} u=0$, i.e. $u$ is constant almost everywhere. 


\subsection{The CLT for unbounded observables}

In order to prove a central limit theorem for the entropy $h\left(T_{\alpha}\right)$ one has to consider the observable $x \mapsto \log \left|T_{\alpha}^{\prime}(x)\right|=-2 \log |x|$, which is not of bounded variation on intervals containing 0 . Therefore, one has to enlarge the space of functions to work with so that it contains such observable, and use some norm which still allows to bound the essential spectral radius of the transfer operator. Such technique will be developed in this section.

The strategy is to use the Ionescu-Tulcea and Marinescu theorem to get a spectral decomposition of the transfer operator, as we did in Section 2.2. This theorem requires a pair of Banach spaces contained in each other such that the operator preserves both. Traditionally, this is achieved by considering the pair $B V(I) \subset L^{1}(I)$. In our case, we will replace the space of functions of bounded variation with newlydefined, larger spaces $B_{K, \delta} \subseteq L^{1}$, which allow for functions with a mild singularity in 0 .

\subsubsection{A new family of Banach spaces}

Fix $\alpha \in(0,1]$. Given a positive integer $K$ and some $0<\delta<1$, let us define the $K, \delta$-norm of a function $f: I_{\alpha} \rightarrow \mathbb{C}$ as

$$
\|f\|_{K, \delta}:=\sup _{k \geq K}\left(k^{-\delta} \operatorname{Var}_{L_{k}} f+\int_{L_{k}}|f(x)| d x\right)
$$

where the $L_{k}$ are a sequence of increasing subintervals of $I_{\alpha}$, namely

$$
L_{k}^{+}:=\overline{\bigcup_{j \geq k} I_{j}^{+}}=\left[\frac{1}{k+\alpha}, \alpha\right] \quad L_{k}^{-}:=\overline{\bigcup_{j \geq k} I_{j}^{-}}=\left[\alpha-1,-\frac{1}{k+\alpha}\right]
$$

and $L_{k}:=L_{k}^{+} \cup L_{k}^{-}$, with $\operatorname{Var}_{L_{k}} f:=\operatorname{Var}_{L_{k}^{+}} f+\operatorname{Var}_{L_{k}^{-}} f$. Let us now define the space $B_{K, \delta}$ of functions of mild growth as

$$
B_{K, \delta}:=\left\{f \in L^{1}: f \text { has a version } g \text { with }\|g\|_{K, \delta}<\infty\right\} .
$$

Let us now establish some basic properties of these spaces. First of all, they are Banach spaces:

Proposition 4.4. For every $K \in \mathbb{N}, 0<\delta<1$, the space $B_{K, \delta}$ endowed with the norm

$$
\|f\|_{K, \delta}:=\inf \left\{\|g\|_{K, \delta}, g=f \text { a.e. }\right\}
$$

is a Banach space.

Proof. This is obviously a normed vector space. Let us prove completeness. If $\left\{f_{n}\right\}$ is a Cauchy sequence, then there exists for every $k$ a function $\bar{f}_{k}$ such that $\left.f_{n}\right|_{L_{k}} \rightarrow \bar{f}_{k}$ in $B V\left(L_{k}\right)$-norm for $n \rightarrow \infty$. Also, by restricting $\left.f_{n}\right|_{L_{k+1}} \rightarrow \bar{f}_{k+1}$ to 
$L_{k}$ one can conclude $\left.\bar{f}_{k+1}\right|_{L_{k}}=\bar{f}_{k}$, hence one can define $f:[\alpha-1, \alpha] \backslash\{0\} \rightarrow \mathbb{C}$ such that $\left.f\right|_{L_{k}}=\bar{f}_{k}$. Now, $\forall \epsilon>0 \exists N \forall m, n \geq N \forall k \geq K$

$$
\left\|f_{m}-f_{n}\right\|_{L^{1}\left(L_{k}\right)}+k^{-\delta} \operatorname{Var}_{L_{k}}\left(f_{m}-f_{n}\right) \leq \epsilon
$$

and by taking the limit for $n \rightarrow \infty$ one has $\left\|f_{m}-f\right\|_{K, \delta} \leq \epsilon$.

Note that $\|f\|_{L^{1}\left(I_{\alpha}\right)} \leq\|f\|_{K, \delta}$, and $B_{K, \delta}$ is a $B V$-module, i.e.

$$
f \in B_{K, \delta}, g \in B V \Rightarrow f g \in B_{K, \delta} .
$$

Another useful property of these spaces is the following:

Lemma 4.5. For $K>\max \left\{\frac{1}{\alpha}, \frac{1}{1-\alpha}\right\}$, with $K \geq 1$ if $\alpha=1$ and $0<\delta<1$ there exists $A>0$ such that $\forall f \in B_{K} . \delta$

$$
|f(x)| \leq \frac{A}{|x|^{\delta}}\|f\|_{K, \delta} \quad \forall x \in[\alpha-1, \alpha] \backslash\{0\} .
$$

Proof. For $f \in B_{K, \delta}, x \in L_{k}^{+} \backslash L_{k-1}^{+}$

$$
|f(x)| \leq|f(x)-f(\alpha)|+|f(\alpha)| \leq \operatorname{Var}_{L_{k}^{+}} f+\sup _{L_{K}^{+}}|f|
$$

and since $x \leq \frac{1}{k-1+\alpha}$

$$
\leq k^{\delta}\|f\|_{K, \delta}+\operatorname{Var}_{L_{K}^{+}} f+\frac{\|f\|_{L^{1}\left(I_{\alpha}\right)}}{m\left(L_{K}^{+}\right)} \leq\left(\left(\frac{1}{|x|}+1\right)^{\delta}+K^{\delta}+\frac{1}{m\left(L_{K}^{+}\right)}\right)\|f\|_{K, \delta} .
$$

Similarly for $x<0$.

Moreover, just as in the case of $B V$, the inclusion $B_{K, \delta} \rightarrow L^{1}$ is compact.

Proposition 4.6. For every $K$ sufficiently large, $\delta>0$, the unit ball

$$
\mathcal{B}=\left\{f \in B_{K, \delta},\|f\|_{K, \delta} \leq 1\right\}
$$

is compact in the $L^{1}$-topology.

Proof. This fact is well-known when you consider $B V$ instead of $B_{K, \delta}$. Now, given $\left\{f_{n}\right\} \subseteq \mathcal{B}$, for any $k$ the sequence of restrictions $\left.f_{n}\right|_{L_{k}}$ sits inside a closed ball in $B V\left(L_{k}\right)$ hence it has a subsequence which converges in $L^{1}\left(L_{k}\right)$ to some $F_{k} \in$ $B V\left(L_{k}\right)$. By refining the subsequence as $k \rightarrow \infty$, one finds a subsequence $f_{n_{l}} \in \mathcal{B}$ such that for every $k,\left.f_{n_{l}}\right|_{L_{k}} \rightarrow F_{k}$ in $L^{1}\left(L_{k}\right)$ and a.e. for $l \rightarrow \infty$. By uniqueness of the limit there exists $F$ such that $\left.F\right|_{L_{k}}=F_{k}$. By lower semicontinuity of total variation, $k^{-\delta} \operatorname{Var}_{L_{k}} F+\|F\|_{L^{1}\left(L_{k}\right)} \leq 1$, so $F \in \mathcal{B}$. We are just left with proving $f_{n_{l}} \rightarrow F$ in $L^{1}\left(I_{\alpha}\right)$ for $l \rightarrow \infty$. By Lemma 4.5

$\int_{I_{\alpha}}\left|f_{n_{l}}-F\right| \leq \int_{I_{\alpha} \backslash L_{k}}\left|f_{n_{l}}\right|+|F|+\int_{L_{k}}\left|f_{n_{l}}-F\right| \leq 2 \int_{I_{\alpha} \backslash L_{k}} \frac{A}{|x|^{\delta}} d x+\int_{L_{k}}\left|f_{n_{l}}-F_{k}\right|$.

The first term tends to 0 as $k \rightarrow \infty$ and the second does so for $l \rightarrow \infty$ as $k$ is fixed. 


\subsubsection{Spectral decomposition in $B_{K, \delta}$}

The goal of this section is to prove a spectral decomposition analogous to Theorem 2.2 in the space $B_{K, \delta}$, namely:

Theorem 4.7. For every $\alpha \in(0,1], 0<\delta<1$ and $K$ sufficiently large, the transfer operator $\Phi_{\alpha}: B_{K, \delta} \rightarrow B_{K, \delta}$ decomposes as

$$
\Phi_{\alpha}=\Pi_{\alpha}+\Psi_{\alpha}
$$

where $\Pi_{\alpha}$ and $\Psi_{\alpha}$ are bounded linear, commuting operators on $B_{K, \delta}, \rho\left(\Psi_{\alpha}\right)<1$ and $\Pi_{\alpha}$ is a projection onto a one-dimensional eigenspace.

The main ingredient to get the spectral decomposition is again a Lasota-Yorke type estimate:

Proposition 4.8. Let $\alpha \in(0,1], 0<\delta<1$. Then there exist $K \in \mathbb{N}, 0<\lambda<1$, $C>0, D>0$ such that

$$
\left\|\Phi_{\alpha}^{n}(f)\right\|_{K, \delta} \leq C \lambda^{n}\|f\|_{K, \delta}+D\|f\|_{L^{1}} \quad \forall f \in B_{K, \delta} .
$$

Proof. First consider the case $\alpha<1$. By formula (2.1) and Lemma 6.1, 4.

$$
\operatorname{Var}_{L_{k}^{+}} \Phi_{\alpha}^{n}(f) \leq \sum_{j \in \mathcal{P}_{n}} \operatorname{Var}_{T_{\alpha}^{n}\left(I_{j}\right) \cap L_{k}^{+}} \frac{f \circ \sigma_{j}}{\left|\left(T_{\alpha}^{n}\right)^{\prime} \circ \sigma_{j}\right|}+2 \sup _{T_{\alpha}^{n}\left(I_{j}\right) \cap L_{k}^{+}}\left|\frac{f \circ \sigma_{j}}{\left|\left(T_{\alpha}^{n}\right)^{\prime} \circ \sigma_{j}\right|}\right|
$$

and by Lemma $6.1,1$. and the fact that $T_{\alpha}^{n}: I_{j} \rightarrow T_{\alpha}^{n}\left(I_{j}\right)$ is a homeomorphism

$$
\begin{aligned}
& \leq \sum_{I_{j} \in \mathcal{P}_{n}} 3 \operatorname{Var}_{I_{j}} \frac{f}{\left|\left(T_{\alpha}^{n}\right)^{\prime}\right|}+\frac{2 \int_{I_{j}}|f(x)| d x}{m\left(T_{\alpha}^{n}\left(I_{j}\right) \cap L_{k}^{+}\right)} \\
& \leq 3 \sum_{I_{j} \in \mathcal{P}_{n}} \operatorname{Var}_{I_{j}}\left(f g_{n, \alpha}\right)+\frac{2\|f\|_{1}}{\inf _{j \in \mathcal{P}_{n}}\left\{m\left(T_{\alpha}^{n}\left(I_{j}\right) \cap L_{k}^{+}\right)\right\}}
\end{aligned}
$$

where the inf is taken over all non-empty intervals. Now, note that by Lemma 6.1 (3) and Proposition 2.1 (2) we have

$$
\sum_{I_{j} \in \mathcal{P}_{n}} \operatorname{Var}_{I_{j}}\left(f g_{n, \alpha}\right) \leq \sum_{I_{j} \in \mathcal{P}_{n}} \operatorname{Var}_{I_{j}} f \sup _{I_{j}} g_{n, \alpha}+\frac{2\|f\|_{1}}{1-\gamma_{\alpha}}
$$


hence we are left with only one term to estimate: in order to do so, we will split the sum in several parts, according to the filtration $L_{k}$ :

$$
\begin{aligned}
\sum_{I_{j} \in \mathcal{P}_{n}} \operatorname{Var}_{I_{j}} f \sup _{I_{j}} g_{n, \alpha} & \leq\left\|g_{n, \alpha}\right\|_{\infty} \sum_{\substack{I_{j} \in \mathcal{P}_{n} \\
I_{j} \subseteq L_{k}}} \operatorname{Var}_{I_{j}} f+\sum_{\substack{h=1 \\
\epsilon= \pm}}^{\infty} \sum_{\substack{I_{j} \in \mathcal{P}_{n} \\
I_{j} \subseteq L_{(h+1) k}^{\epsilon} \backslash L_{h k}^{\epsilon}}} \operatorname{Var}_{I_{j}} f \sup _{I_{j}} g_{n, \alpha} \\
& \leq \gamma_{\alpha}^{n} \operatorname{Var}_{L_{k}} f+\sum_{\substack{h=1 \\
\epsilon= \pm}}^{\infty} \operatorname{Var}_{L_{(h+1) k}^{\epsilon}} f \sup _{L_{(h+1) k}^{\epsilon} \backslash L_{h k}^{\epsilon}} g_{n, \alpha} \\
& \leq \gamma_{\alpha}^{n} k^{\delta}\|f\|_{K, \delta}+\sum_{h=1}^{\infty}\|f\|_{K, \delta}[(h+1) k]^{\delta} \sup _{L_{(h+1) k} \backslash L_{h k}} g_{1, \alpha}
\end{aligned}
$$

and since $L_{(h+1) k} \backslash L_{h k}=\left[-\frac{1}{h k+\alpha},-\frac{1}{(h+1) k+\alpha}\right) \cup\left(\frac{1}{(h+1) k+\alpha}, \frac{1}{h k+\alpha}\right]$

$$
\leq\|f\|_{K, \delta} k^{\delta}\left(\gamma_{\alpha}^{n}+\sum_{h=1}^{\infty} \frac{(h+1)^{\delta}}{h^{2} k^{2}}\right) \leq\|f\|_{K, \delta} k^{\delta}\left(\gamma_{\alpha}^{n}+\frac{M}{K^{2}}\right)
$$

for some universal constant $M$ for all $k \geq K$. The same estimate holds for $\operatorname{Var}_{L_{k}^{-}} \Phi_{\alpha}^{n}(f)$. Moreover, for fixed $n$ and $\alpha$ the set $\left\{T_{\alpha}^{n}\left(I_{j}\right) \mid I_{j} \in \mathcal{P}_{n}\right\}$ is finite, and since $L_{k}^{+}$and $L_{k}^{-}$are increasing sequences of intervals, $\inf \left\{m\left(T_{\alpha}^{n}\left(I_{j}\right) \cap L_{k}^{ \pm}\right)\right.$: $\left.I_{j} \in \mathcal{P}_{n}, k \geq K\right\}$ is bounded below by a positive constant, and for every $\alpha$ one can choose $n$ and $K$ such that $\lambda:=6\left(\gamma_{\alpha}^{n}+\frac{M}{K^{2}}\right)<1$. By combining all these estimates, there exists a constant $D$ such that

$$
\left\|\Phi_{\alpha}^{n}(f)\right\|_{K, \delta} \leq \lambda\|f\|_{K, \delta}+D\|f\|_{1} \quad \forall f \in B_{K, \delta}
$$

and by iteration the claim follows. The case $\alpha=1$ follows similarly; in this case $\left\|g_{1, \alpha}\right\|_{\infty}=1$, but Proposition 2.1 is replaced by

$$
\left\|g_{n, \alpha}\right\|_{\infty} \leq 4\left(\frac{\sqrt{5}-1}{2}\right)^{2 n-4} \quad\left\|g_{n, \alpha}^{\prime}\right\|_{\infty} \leq 2 .
$$

Proof of Theorem 4.7. By Propositions 4.6 and 4.8, the transfer operators $\Phi_{\alpha}$ acting on $B_{K, \delta}$ satisify the hypotheses of Ionescu-Tulcea and Marinescu's theorem [6], hence we have a spectral decomposition of $\Phi_{\alpha}$ with a finite number of spectral projectors onto eigenvalues of unit modulus. Moreover, mixing of $T_{\alpha}$ still implies there is only one eigenvalue of modulus one and its eigenspace is one-dimensional.

Note that since $B V\left(I_{\alpha}\right) \subseteq B_{K, \delta}$, the invariant density $\rho_{\alpha}$ previously obtained is still a fixed point of $\Phi_{\alpha}$, hence $\Pi_{\alpha}$ is nothing but projection onto $\mathbb{C} \rho_{\alpha}$. 


\subsubsection{End of the proof}

The proof of Theorem 1.2 now follows from a standard application of the martingale central limit theorem. We will refer to the version given in [18, Theorem 2.11]. In order to adapt it to our situation, we need the following two lemmas:

Lemma 4.9. Let $\alpha \in(0,1], 0<\delta<\frac{1}{2}$, and $K$ such that Theorem 4.7 holds, and consider $f \in B_{K, \delta}$ with $\int_{I_{\alpha}} f d \mu_{\alpha}=0$. Denote by $\mathcal{F}_{0}$ the Borel $\sigma$-algebra on $I_{\alpha}$ and $\mathcal{F}_{n}:=T_{\alpha}^{-n}\left(\mathcal{F}_{0}\right)$. Then

$$
\sum_{n=0}^{\infty}\left\|\mathbb{E}\left(f \mid \mathcal{F}_{n}\right)\right\|_{L^{2}\left(\mu_{\alpha}\right)}<+\infty
$$

Proof.

$$
\begin{aligned}
\left\|\mathbb{E}\left(f \mid \mathcal{F}_{n}\right)\right\|_{L^{2}\left(\mu_{\alpha}\right)} & =\sup \left\{\int_{I_{\alpha}}\left(\psi \circ T_{\alpha}^{n}\right) f d \mu_{\alpha}: \psi \in L^{2}\left(\mu_{\alpha}\right),\|\psi\|_{L^{2}\left(\mu_{\alpha}\right)}=1\right\} \\
& =\sup \left\{\int_{I_{\alpha}} \psi \Phi_{\alpha}^{n}\left(f \rho_{\alpha}\right) d x: \psi \in L^{\infty}\left(\mu_{\alpha}\right),\|\psi\|_{L^{2}\left(\mu_{\alpha}\right)}=1\right\} \\
& \leq \frac{\left\|\Phi_{\alpha}^{n}\left(f \rho_{\alpha}\right)\right\|_{L^{2}(d x)}}{\sqrt{\inf \rho_{\alpha}}} .
\end{aligned}
$$

Now, by Lemma 4.5 and since $0<\delta<\frac{1}{2},\left\|\Phi_{\alpha}^{n}\left(f \rho_{\alpha}\right)\right\|_{L^{2}(d x)} \leq C\left\|\Phi_{\alpha}^{n}\left(f \rho_{\alpha}\right)\right\|_{K, \delta}$, and by Theorem $4.7 \Phi_{\alpha}^{n}\left(f \rho_{\alpha}\right)=\Psi^{n}\left(f \rho_{\alpha}\right)$ goes to 0 exponentially fast in $B_{K, \delta^{-}}$ norm as $n \rightarrow \infty$.

Lemma 4.10. Let $f \in B_{K, \delta}$ be real-valued, non-constant and such that $\int_{I_{\alpha}} f d \mu_{\alpha}=$ 0 . Then there exists no function $u \in B_{K, \delta}$ such that

$$
f=u-u \circ T_{\alpha} \quad \mu_{\alpha}-\text { a.e. }
$$

Proof. Notice that $\mu_{\alpha}$ and the Lebesgue measure are absolutely continuous with respect to each other, hence measure zero sets are the same. Suppose there exists $u$ which satisfies the equation; then, $u \circ T_{\alpha}$ belongs to $B_{K, \delta}$. However,

$\operatorname{Var}_{L_{k}^{+}}\left(u \circ T_{\alpha}\right) \geq \sum_{\substack{I_{j} \text { full } \\ j \leq k}} \operatorname{Var}_{I_{j}}\left(u \circ T_{\alpha}\right)=\sum_{\substack{I_{j} \text { full } \\ j \leq k}} \operatorname{Var}_{(\alpha-1, \alpha)} u=\left(k-j_{\min }\right) \operatorname{Var}_{(\alpha-1, \alpha)} u$.

On the other hand, $\operatorname{Var}_{L_{k}^{+}}\left(u \circ T_{\alpha}\right) \leq k^{\delta}\left\|u \circ T_{\alpha}\right\|_{K, \delta}$ with $\delta<1$, which contradicts the previous estimate unless $\operatorname{Var}_{(\alpha-1, \alpha)} u=0$, i.e. $u$ is constant almost everywhere.

Proof of Theorem 1.2. We can assume $\int_{I_{\alpha}} f d \mu_{\alpha}=0$. By [18, Theorem 2.11] and Lemma 4.9, the claim follows unless there exists $u \in L^{2}\left(\mu_{\alpha}\right)$ such that

$$
f=u-u \circ T_{\alpha} \quad \mu_{\alpha}-\text { a.e. }
$$


If there exists such a $u$, one can assume that $\int u d \mu_{\alpha}=0$, and then, by the proof of [18, Theorem 2.11], $u$ is given by

$$
u=-\sum_{j=1}^{\infty} \frac{\Phi_{\alpha}^{j}\left(f \rho_{\alpha}\right)}{\rho_{\alpha}}
$$

where convergence of the series is in $L^{2}\left(\mu_{\alpha}\right)$. By the spectral decomposition, $\sum_{j} \Phi^{j}\left(f \rho_{\alpha}\right)$ converges also in $B_{K, \delta} \subseteq L^{2}\left(\mu_{\alpha}\right)$. Moreover, since $\rho_{\alpha}$ is in $B V$ and is bounded from below, then $\frac{1}{\rho_{\alpha}}$ is in $B V$. Thus, $u$ lies in $B_{K, \delta}$, and this contradicts Lemma 4.10 unless $f$ is constant.

Now, the function $x \mapsto \log |x|$ belongs to every $B_{K, \delta}$, therefore we have:

Corollary 4.11. For every $\alpha \in(0,1]$, the Birkhoff averages for the observable $\log \left|T_{\alpha}^{\prime}(x)\right|=-2 \log |x|$ distribute normally around the value $h\left(T_{\alpha}\right)$.

\section{Stability of the standard deviation}

Having established the convergence of Birkhoff sums to a Gaussian distribution, we are now interested in analyzing how the standard deviation of this Gaussian varies when $\alpha$ varies. The question is motivated by the numerical simulations in [2, Section 2]. We prove the following:

Theorem 5.1. Let $f:(-1,1) \rightarrow \mathbb{R}$ be of class $C^{1}$. For every $\alpha \in(0,1)$ let us consider the variance

$$
\sigma_{\alpha}^{2}:=\lim _{n \rightarrow \infty} \int_{I_{\alpha}}\left(\frac{S_{n} f-n \int_{I_{\alpha}} f d \mu_{\alpha}}{\sqrt{n}}\right)^{2} d \mu_{\alpha} .
$$

Then for every $\underline{\alpha} \in(0,1)$ we have

$$
\lim _{\alpha \rightarrow \underline{\alpha}} \sigma_{\alpha}^{2}=\sigma_{\underline{\alpha}}^{2}
$$

The variance $\sigma_{\alpha}^{2}$ of the limit distribution is the second derivative of the eigenvalues $\lambda_{0}(\theta)$ of the perturbed transfer operators $\left\{\Phi_{\alpha, f, \theta}\right\}$ (see the discussion in Section 4.1, and in particular equation (2) after Proposition 4.2). In order to prove the theorem, we will prove uniform convergence in $\alpha$ of the eigenvalues, via application

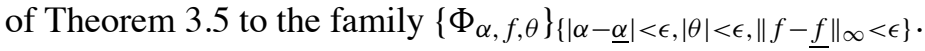

Hypothesis (1) of Theorem 3.5 is easily proved:

Lemma 5.2. For any $C>0$ there exists $M>0$ such that

$$
\left\|\Phi_{\alpha, f, \theta}^{n}\right\|_{1} \leq M^{n} \quad \forall n \in \mathbb{N} \forall \alpha \in(0,1) \forall|\theta|<C
$$

for every $f \in L^{\infty}\left(I_{\alpha}\right)$ s.t $\|f\|_{\infty} \leq C$. 
Proof. For $g \in B V$

$\left\|\Phi_{\alpha, f, \theta}^{n}(g)\right\|_{1}=\left\|\Phi_{\alpha}^{n}\left(e^{\theta S_{n} f} g\right)\right\|_{1} \leq\left\|e^{\theta S_{n} f} g\right\|_{1} \leq\left\|e^{\theta S_{n} f}\right\|_{\infty}\|g\|_{1} \leq e^{n|\operatorname{Re} \theta|\|f\|_{\infty}}\|g\|_{1}$

where we used the fact that the unperturbed operators have unit norm on $L^{1}$.

Hypothesis (4) follows directly from Lemma 3.9; the precise statement, the proof of which we omit, is the following:

Lemma 5.3. Let $\underline{\alpha} \in(0,1)$. Then there exist $\epsilon, C>0$ such that for any $f, \underline{f} \in$ $B V([0,1])$ such that $\|f-\underline{f}\|_{\infty}<\epsilon, \forall|\theta|<\epsilon, \forall|\alpha-\underline{\alpha}|<\epsilon$

$$
\left\|\Phi_{\underline{\alpha}, \underline{f}, \theta}-\Phi_{\alpha, f, \theta}\right\| \leq C\left(|\alpha-\underline{\alpha}|^{1 / 2}+\|f-\underline{f}\|_{\infty}\right) .
$$

We now check condition (2) using the estimates in Section 3.1 to get a Lasota-Yorke inequality which is uniform in both $\alpha$ and $\theta$.

Proposition 5.4. Let $\underline{\alpha} \in(0,1)$. There exist $0<\lambda<1, \epsilon, C_{2}, C_{3}$ such that

$$
\operatorname{Var}_{I_{\alpha}} \Phi_{\alpha, f, \theta}^{n}(g) \leq C_{2} \lambda^{n} \operatorname{Var}_{I_{\alpha}} g+C_{3}\|g\|_{1} \quad \forall n \in \mathbb{N}
$$

for every $\alpha \in(\underline{\alpha}-\epsilon, \underline{\alpha}+\epsilon)$, for every $|\theta|<\epsilon$ and for every $f \in C^{1}\left(I_{\alpha}\right)$ with $\|f\|_{C^{1}} \leq 1$.

Proof. Let us fix $g \in B V$. We have

$$
\begin{aligned}
\operatorname{Var}_{I_{\alpha}} \Phi_{\alpha, f, \theta}^{n}(g) & =\operatorname{Var}_{I_{\alpha}} \Phi_{\alpha}^{n}\left(e^{\theta S_{n} f} g\right) \leq \operatorname{Var}_{I_{\alpha}}\left(e^{\theta S_{n} f} g \cdot g_{n, \alpha}\right) \\
& =\sum_{j \in \mathcal{P}_{n}} \operatorname{Var}_{I_{j}}\left(e^{\theta S_{n} f} g \cdot g_{n, \alpha}\right) .
\end{aligned}
$$

Note that, since $\left.g_{n, \alpha}\right|_{\partial I_{j}}=0$,

$$
\operatorname{Var}_{I_{j}}\left(e^{\theta S_{n} f} g g_{n, \alpha}\right) \leq \operatorname{Var}_{I_{j}}\left(e^{\theta S_{n} f} g g_{n, \alpha}\right)+e^{n|\theta|\|f\|_{\infty}} \operatorname{Var}_{\overline{I_{j}}}\left(g g_{n, \alpha}\right) \text {. }
$$

Now, by Lemma $6.1,3$

$\operatorname{Var}_{I_{j}}\left(e^{\theta S_{n} f} g_{n, \alpha} g\right)=\operatorname{Var}_{I_{j}} \frac{e^{\theta S_{n} f} g}{\left|\left(T_{\alpha}^{n}\right)^{\prime}\right|} \leq \sup _{I_{j}}\left|\left(\frac{e^{\theta S_{n} f}}{\left(T_{\alpha}^{n}\right)^{\prime}}\right)^{\prime}\right| \int_{I_{j}}|g|+\sup _{I_{j}}\left|\frac{e^{\theta S_{n} f}}{\left(T_{\alpha}^{n}\right)^{\prime}}\right| \operatorname{Var}_{I_{j}} g$

and by expanding the derivative

$$
\begin{aligned}
\left(\frac{e^{\theta S_{n} f}}{\left(T_{\alpha}^{n}\right)^{\prime}}\right)^{\prime} & =\frac{\left(e^{\theta S_{n} f}\right)^{\prime}}{\left(T_{\alpha}^{n}\right)^{\prime}}+\left(\frac{1}{\left(T_{\alpha}^{n}\right)^{\prime}}\right)^{\prime} e^{\theta S_{n} f} \\
& =\frac{e^{\theta S_{n} f} \theta \sum_{k=0}^{n-1}\left(f^{\prime} \circ T_{\alpha}^{k}\right)\left(T_{\alpha}^{k}\right)^{\prime}}{\left(T_{\alpha}^{n}\right)^{\prime}}+\left(\frac{1}{\left(T_{\alpha}^{n}\right)^{\prime}}\right)^{\prime} e^{\theta S_{n} f} \\
& =e^{\theta S_{n} f}\left[\theta \sum_{k=0}^{n-1} \frac{\left(f^{\prime} \circ T_{\alpha}^{k}\right)}{\left[\left(T_{\alpha}^{n-k}\right)^{\prime} \circ T_{\alpha}^{k}\right]}+\left(\frac{1}{\left(T_{\alpha}^{n}\right)^{\prime}}\right)^{\prime}\right] \\
& \leq e^{n|\theta|\|f\|_{\infty}} \frac{|\theta|\left\|f^{\prime}\right\|_{\infty}+2}{1-\gamma_{\alpha}} .
\end{aligned}
$$


Moreover, by the estimates of Proposition 3.1 (equation (3.2)), for each $\underline{\alpha} \in(0,1)$ and each $n$ there exist $\eta, D$ such that

$$
\operatorname{Var}_{I_{\alpha}}\left(g g_{n, \alpha}\right) \leq(2 n+4) \gamma_{\alpha}^{n} \operatorname{Var}_{I_{\alpha}} g+D\|g\|_{1} \quad \forall \alpha \in(\underline{\alpha}-\eta, \underline{\alpha}+\eta) ;
$$

hence by combining all the previous estimates

$\operatorname{Var}_{I_{\alpha}} \Phi_{\alpha, f, \theta}^{n}(g) \leq(2 n+5) e^{n|\theta|\|f\|_{\infty}} \gamma_{\alpha}^{n} \operatorname{Var}_{I_{\alpha}} g+e^{n|\theta|\|f\|_{\infty}}\left(\frac{|\theta|\left\|f^{\prime}\right\|_{\infty}+2}{1-\gamma_{\alpha}}+D\right)\|g\|_{1}$

and the claim follows by choosing some $n$ large enough and iterating.

Remark 5.5. Notice that this is the only place where we need $f \in C^{1}$. This is because, if $f \in B V, e^{\theta S_{n} f}$ will not in general be of bounded variation.

We are now ready to draw consequences for the spectral decomposition: let us denote by $\lambda_{\alpha, f}(\theta)$ the eigenvalue of $\Phi_{\alpha, f, \theta}$ which is closest to 1 .

Lemma 5.6. Let $\underline{\alpha} \in(0,1)$ and suppose we have a family $\left\{f_{\alpha}\right\}_{\alpha \in(0,1)}$ of functions $f_{\alpha}:[0,1] \rightarrow \mathbb{R}$ of class $C^{1}$ for every $\alpha$ and such that

- $\left\|f_{\alpha}-f_{\underline{\alpha}}\right\|_{\infty} \rightarrow 0$ for $\alpha \rightarrow \underline{\alpha}$,

$-\sup _{\alpha \in(0,1)}\left\|f_{\alpha}^{\prime}\right\|_{\infty}<\infty$.

Then there exists $\epsilon>0$ such that $\lambda_{\alpha, f_{\alpha}}(\theta)$ converges to $\lambda_{\underline{\alpha}, f_{\underline{\alpha}}}(\theta)$ on $|\theta|<\epsilon$ uniformly in $\theta$ as $\alpha \rightarrow \underline{\alpha}$.

Proof. Let us fix $r \in\left(\lambda_{0}, 1\right)$ and $\delta$ such that $0<\delta<\frac{1-r}{2}$. Then the projectors

$$
\Pi_{\alpha, f_{\alpha}, \theta}:=\frac{1}{2 \pi i} \oint_{\partial B(1, \delta)}\left(z-\Phi_{\alpha, f_{\alpha}, \theta}\right)^{-1} d z
$$

are defined for $|\alpha-\underline{\alpha}|<\epsilon$ and $|\theta|<\epsilon$ for some $\epsilon$ and for $\delta$ sufficiently small; moreover $\operatorname{rank}\left(\Pi_{\alpha, f_{\alpha}, \theta}\right)=\operatorname{rank}\left(\Pi_{\underline{\alpha}, f_{\alpha}, 0}\right)=1[8$, Corollary 3$]$ so they are all projections on the 1-dimensional eigenspace relative to the eigenvalue which is closest to 1. By Dunford calculus we also have

$$
\lambda_{\alpha, f_{\alpha}}(\theta) \Pi_{\alpha, f_{\alpha}, \theta}=\Phi_{\alpha, f_{\alpha}, \theta} \Pi_{\alpha, f_{\alpha}, \theta}=\frac{1}{2 \pi i} \oint_{\partial B(1, \delta)} z\left(z-\Phi_{\alpha, f_{\alpha}, \theta}\right)^{-1} d z .
$$

By Theorem 3.5 and Proposition 5.3 there exists $C$ such that for $|\underline{\alpha}-\alpha|<\epsilon$ and $|\theta|<\epsilon$

$$
\left\|\left(z-\Phi_{\alpha, f_{\alpha}, \theta}\right)^{-1}-\left(z-\Phi_{\underline{\alpha}, f_{\underline{\alpha}}, \theta}\right)^{-1}\right\| \leq C\left(|\alpha-\underline{\alpha}|^{1 / 2}+\left\|f_{\alpha}-f_{\underline{\alpha}}\right\|_{\infty}\right)^{\eta}
$$

with $\eta>0$ fixed by Theorem 3.5 so by equations (5.1) and (5.2)

$$
\left|\lambda_{\alpha}(\theta)-\lambda_{\underline{\alpha}}(\theta)\right|=O\left(|\alpha-\underline{\alpha}|^{1 / 2}+\left\|f_{\alpha}-f_{\underline{\alpha}}\right\|_{\infty}\right)^{\eta}
$$

uniformly in $\theta$ as $\alpha \rightarrow \underline{\alpha}$. 
Proof of Theorem 5.1. Let $f_{\alpha}:[0,1] \rightarrow \mathbb{R}$ be $f_{\alpha}(x):=f(x+\alpha-1)-\int_{\alpha-1}^{\alpha} f d \mu_{\alpha}$. Since $\tilde{\rho}_{\alpha} \rightarrow \tilde{\rho}_{\underline{\alpha}}$ in $L^{1}$ and $f(x+\alpha-1) \rightarrow f(x+\underline{\alpha}-1)$ in $L^{\infty}$, we have $\int_{\alpha-1}^{\alpha} f d \mu_{\alpha} \rightarrow \int_{\underline{\alpha}-1}^{\alpha} f d \mu_{\underline{\alpha}}$, and the family $\left\{f_{\alpha}\right\}$ satisfies the hypotheses of Lemma 5.6, therefore $\lambda_{\alpha, f_{\alpha}}(\theta)$ converges uniformly in a nbd of $\theta=0$ to $\lambda_{\underline{\alpha}}, f_{\underline{\alpha}}(\theta)$. Since all $\lambda_{\alpha, f_{\alpha}}(\theta)$ are analytic in $\theta$ you also have convergence of all derivatives, in particular $\lambda_{\alpha, f_{\alpha}}^{\prime \prime}(0) \rightarrow \lambda_{\underline{\alpha}, f_{\underline{\alpha}}}^{\prime \prime}(0)$. We now note that $\int_{0}^{1} f_{\alpha}(x) \tilde{\rho}_{\alpha}(x) d x=0$, which implies, as we have seen in Section 2.2, that $\lambda_{\alpha, f_{\alpha}}^{\prime \prime}(0)=\sigma_{\alpha}^{2}$.

\section{Appendix}

Let us recall a few well-known properties of functions with total variation:

Lemma 6.1. Let $I \subseteq \mathbb{R}$ be a bounded interval, $J \subseteq I$ a subinterval and $f$ of bounded variation. Then:

(1) $\sup _{x \in J}|f(x)| \leq \operatorname{Var}_{J} f+\frac{1}{m(J)} \int_{J}|f(x)| d x$;

(2) If $g \in B V(J)$ then

$$
\operatorname{Var}_{J}(f g) \leq \sup _{x \in J}|f(x)| \operatorname{Var}_{J} g+\sup _{x \in J}|g(x)| \operatorname{Var}_{J} f
$$

(3) If $g$ is of class $C^{1}$ on $J$ then

$$
\operatorname{Var}_{J}(f g) \leq \operatorname{Var}_{J} f \sup _{x \in J}|g(x)|+\sup _{x \in J}\left|g^{\prime}(x)\right| \int_{J}|f(x)| d x ;
$$

(4) $\operatorname{Var}_{I}\left(f \chi_{J}\right) \leq \operatorname{Var}_{J} f+2 \sup _{J}|f|$.

Let us also prove the basic properties of $T_{\alpha}$ mentioned in Section 2.1.

Proof of Proposition 2.1.

(1) $\sup _{j \in \mathcal{P}_{1}} \sup _{x \in I_{j}} \frac{1}{\left|\left(T_{\alpha}\right)^{\prime}(x)\right|}=\sup _{x \in[\alpha-1, \alpha]} x^{2} \leq \max \left\{\alpha^{2},(\alpha-1)^{2}\right\}$. The case for $n>1$ follows from the chain rule for derivatives.

(2) Let $K_{n}:=\sup _{j \in \mathcal{P}_{n}} \sup _{x \in I_{j}}\left|g_{n, \alpha}^{\prime}(x)\right|$. For $n=1$,

$$
K_{1}=\sup _{j \in \mathcal{P}_{1}} \sup _{x \in I_{j}}\left|\left(\frac{1}{\left(T_{\alpha}\right)^{\prime}}\right)^{\prime}(x)\right|=\sup _{x \in[\alpha-1, \alpha]} 2|x| \leq 2 .
$$

Now,

$$
\begin{aligned}
\left(T_{\alpha}^{n+1}\right)^{\prime}(x) & =\left(T_{\alpha}^{n}\right)^{\prime}\left(T_{\alpha}(x)\right) T_{\alpha}^{\prime}(x) \\
\left(T_{\alpha}^{n+1}\right)^{\prime \prime}(x) & =\left(T_{\alpha}^{n}\right)^{\prime \prime}\left(T_{\alpha}(x)\right)\left[T_{\alpha}^{\prime}(x)\right]^{2}+\left(T_{\alpha}^{n}\right)^{\prime}\left(T_{\alpha}(x)\right) T_{\alpha}^{\prime \prime}(x) .
\end{aligned}
$$


For every $x$ in the interior of some interval $I_{j} \in \mathcal{P}_{n+1}$,

$$
\begin{aligned}
\left|\frac{\left(T_{\alpha}^{n+1}\right)^{\prime \prime}(x)}{\left[\left(T_{\alpha}^{n+1}\right)^{\prime}(x)\right]^{2}}\right| & \leq\left|\frac{\left(T_{\alpha}^{n}\right)^{\prime \prime}\left(T_{\alpha}(x)\right)\left(T_{\alpha}^{\prime}(x)\right)^{2}}{\left[\left(T_{\alpha}^{n}\right)^{\prime}\left(T_{\alpha}(x)\right) T_{\alpha}^{\prime}(x)\right]^{2}}+\frac{\left(T_{\alpha}^{n}\right)^{\prime}\left(T_{\alpha}(x)\right) T_{\alpha}^{\prime \prime}(x)}{\left[\left(T_{\alpha}^{n}\right)^{\prime}\left(T_{\alpha}(x)\right) T_{\alpha}^{\prime}(x)\right]^{2}}\right| \\
& \leq\left|\frac{\left(T_{\alpha}^{n}\right)^{\prime \prime}\left(T_{\alpha}(x)\right)}{\left[\left(T_{\alpha}^{n}\right)^{\prime}\left(T_{\alpha}(x)\right)\right]^{2}}\right|+\left|\frac{T_{\alpha}^{\prime \prime}(x)}{\left[T_{\alpha}^{\prime}(x)\right]^{2}}\right| \frac{1}{\left|\left(T_{\alpha}^{n}\right)^{\prime}\left(T_{\alpha}(x)\right)\right|} \\
& \leq K_{n}+K_{1} \gamma_{\alpha}^{n}
\end{aligned}
$$

hence $K_{n+1} \leq K_{n}+2 \gamma_{\alpha}^{n}$ and by induction $K_{n} \leq \sum_{k=0}^{n-1} 2 \gamma_{\alpha}^{k} \leq \frac{2}{1-\gamma_{\alpha}}$.

(3) By induction on $n$ : let $I_{j_{M}}^{-}$be the interval of the partition $\mathcal{P}_{1}$ which contains $\alpha-1$ and $I_{j_{m}}^{+}$be the one which contains $\alpha$.

For $n=1, T_{\alpha}\left(I_{j}\right)=I_{\alpha}$ for $I_{j} \neq I_{j_{M}}^{-}, I_{j_{m}}^{+}$, hence

$$
\left\{T_{\alpha}\left(I_{j}\right) \mid I_{j} \in \mathcal{P}_{1}\right\} \subseteq\left\{I_{\alpha}, T_{\alpha}\left(I_{j_{M}}^{-}\right), T_{\alpha}\left(I_{j_{m}}^{+}\right)\right\} .
$$

Let $n>1$; consider an element of the partition $\mathcal{P}_{n+1}$, which will be of the form $I_{j_{0}} \cap T^{-1}\left(I_{j_{1}}\right) \cap \cdots \cap T^{-n}\left(I_{j_{n}}\right) \neq \emptyset$, with $I_{j_{0}}, \ldots, I_{j_{n}} \in \mathcal{P}_{1}$. If we let $L:=I_{j_{1}} \cap \cdots \cap T^{-(n-1)}\left(I_{j_{n}}\right)$, we have $L \neq \emptyset$ and $L \in \mathcal{P}_{n}$. Moreover, one verifies that

$$
T_{\alpha}^{n+1}\left(I_{j_{0}} \cap T_{\alpha}^{-1}\left(I_{j_{1}}\right) \cap \cdots \cap T_{\alpha}^{-n}\left(I_{j_{n}}\right)\right) \subseteq T_{\alpha}^{n}\left(I_{j_{1}} \cap \cdots \cap T_{\alpha}^{-(n-1)}\left(I_{j_{n}}\right)\right)=T_{\alpha}^{n}(L) .
$$

At this point we have two cases:

- If $T_{\alpha}\left(I_{j_{0}}\right) \supseteq L$ then

$$
T_{\alpha}^{n+1}\left(I_{j_{0}} \cap T_{\alpha}^{-1}\left(I_{j_{1}}\right) \cap \cdots \cap T_{\alpha}^{-n}\left(I_{j_{n}}\right)\right)=T_{\alpha}^{n}(L) ;
$$

- Otherwise we have $T_{\alpha}\left(I_{j_{0}}\right) \nsupseteq L$ but $T_{\alpha}\left(I_{j_{0}}\right) \cap L \neq \emptyset$ (if the intersection is empty, so it is the interval we started with); since $T_{\alpha}\left(I_{j}\right)=I$ for $I_{j} \neq$ $I_{j_{M}}^{-}, I_{j_{m}}^{+}$, this implies $I_{j_{0}} \in\left\{I_{j_{M}}^{-}, I_{j_{m}}^{+}\right\}$. Moreover, because $T_{\alpha}\left(I_{j_{M}}^{-}\right)$and $T_{\alpha}\left(I_{j_{m}}^{+}\right)$are intervals with supremum equal to $\alpha$, there exists at most one interval $I_{\mu}$ of the partition $\mathcal{P}_{n}$ such that $T_{\alpha}\left(I_{j_{m}}^{+}\right) \cap I_{\mu} \neq \emptyset$ and $T_{\alpha}\left(I_{j_{m}}^{+}\right) \nsupseteq$ $I_{\mu}$; in the same way there exists only one interval $I_{\nu}$ of the partition $\mathcal{P}_{n}$ such that $T_{\alpha}\left(I_{j_{M}}^{-}\right) \cap I_{\nu} \neq \emptyset$ and $T_{\alpha}\left(I_{j_{M}}^{-}\right) \nsupseteq I_{\nu}$, therefore either $L=I_{\mu}$ or $L=I_{\nu}$.

In conclusion $\left\{T_{\alpha}^{n+1}\left(I_{j}\right) \mid I_{j} \in \mathcal{P}_{n+1}\right\}$ is contained in

$$
\left\{T_{\alpha}^{n}\left(I_{j}\right) \mid I_{j} \in \mathcal{P}_{n}\right\} \cup T_{\alpha}^{n+1}\left(I_{j_{m}}^{+} \cap T_{\alpha}^{-1}\left(I_{\mu}\right)\right) \cup T_{\alpha}^{n+1}\left(I_{j_{M}}^{-} \cap T_{\alpha}^{-1}\left(I_{\nu}\right)\right)
$$

hence at every step the cardinality can only increase by at most 2 . 
(4) Recall that

$$
g_{1, \alpha}(x):= \begin{cases}x^{2} & \text { if } x \text { belongs to some } I_{j} \\ 0 & \text { otherwise }\end{cases}
$$

hence the claim follows from summability of the series $\sum \frac{1}{k^{2}}$.

\section{References}

[1] A. BRoISE, Transformations dilatantes de l'intervalle et théorèmes limites, In: "Études spectrales d'opérateurs de transfert et applications", A. Broise, F. Dal'Bo, M. Peigné (eds.) Astérisque, Vol. 238, Soc. Math. France, Paris, 1996, 1-109.

[2] C. Carminati, S. Marmi, A. Profeti and G. Tiozzo, The entropy of $\alpha$-continued fractions: numerical results, Nonlinearity 23 (2010), 2429-2456.

[3] C. CARMinati and G. TIOzZo, A canonical thickening of $\mathbb{Q}$ and the entropy of $\alpha$-continued fraction transformations, Ergodic Theory Dynam. Systems 32 (2012), 1249-1269.

[4] C. CARMinati and G. TIOzZo, Tuning and plateaux for the entropy of $\alpha$-continued fractions, Nonlinearity 26 (2013), 1049-1070.

[5] H. Hennion, Sur un théorème spectral et son application aux noyaux lipchitziens, Proc. Amer. Math. Soc. 118 (1993), 627-634.

[6] C. T. Ionescu-TulceA and G. Marinescu, Théorie ergodique pour des classes d'opérations non complètement continues, Ann. of Math. 52 (1950), 140-147.

[7] G. Keller, Stochastic stability in some chaotic dynamical systems, Monatsh. Math. 94 (1982), 313-333.

[8] G. KelleR and C. LiVerani, Stability of the spectrum for transfer operators, Ann. Scuola Norm. Sup. Pisa Cl. Sci. (4) 28 (1999), 141-152.

[9] S. KATOK and I. UGARCOVICI, Structure of attractors for $(a, b)$-continued fraction transformations, J. Mod. Dyn. 4 (2010), 637-691.

[10] C. KraAikamp, T. Schmidt and W. Steiner, Natural extensions and entropy of $\alpha$ continued fractions, Nonlinearity 25 (2012), 2207-2243.

[11] L. LUZZI and S. MARMI, On the entropy of Japanese continued fractions, Discrete Contin. Dyn. Syst. 20 (2008), 673-711.

[12] A. CASSA, P. MousSA and S. MARMI, Continued fractions and Brjuno functions, J. Comput. Appl. Math. 105 (1995), 403-415.

[13] H. NAKADA, Metrical theory for a class of continued fraction transformations and their natural extensions, Tokyo J. Math. 4 (1981), 399-426.

[14] H. NAKADA and R. NATSUI, The non-monotonicity of the entropy of $\alpha$-continued fraction transformations, Nonlinearity 21 (2008), 1207-1225.

[15] V. A. RohLIn, Exact endomorphisms of a Lebesgue space, Izv. Akad. Nauk SSSR Ser. Mat. 25 (1961), 499-530; English translation: Amer. Math. Soc. Transl. (2) 39 (1964), 1-36.

[16] M. RYCHLIK, Bounded variation and invariant measures, Studia Math. 76 (1983), 69-80.

[17] F. SCHWEIGER, "Ergodic Theory of Fibred Systems and Metric Number Theory", Oxford Science Publications, Clarendon Press, Oxford, 1995.

[18] M. Viana, "Stochastic Dynamics of Deterministic Systems", Lecture Notes XXI, Braz. Math. Colloq. IMPA, Rio de Janeiro, 1997. 
THE ENTROPY OF NAKADA's $\alpha$-CONTINUED FRACTIONS: ANALYTICAL RESULTS 1037

[19] R. ZWEIMÜLLER, Ergodic structure and invariant densities of non-Markovian interval maps with indifferent fixed points, Nonlinearity 11 (1998), 1263-1276.

Yale University

10 Hillhouse Avenue

New Haven CT 06511 USA

giulio.tiozzo@yale.edu 